\title{
Dynamical exploration of the repertoire of brain networks at rest is modulated by psilocybin
}

\author{
Louis-David Lord ${ }^{1}$, Paul Expert ${ }^{2,3}$, Selen Atasoy ${ }^{1}$, Leor Roseman ${ }^{4}$, Kristina Rapuano ${ }^{5}$, Renaud \\ Lambiotte $^{6}$, David J. Nutt ${ }^{4}$, Gustavo Deco ${ }^{7,8}$, Robin L. Carhart-Harris ${ }^{4}$, Morten L. Kringelbach ${ }^{1,9,10}$ \\ \& Joana Cabral ${ }^{1,9,11}$
}

${ }^{1}$ Department of Psychiatry, University of Oxford, UK

${ }^{2}$ Centre for Mathematics of Precision Healthcare, Imperial College London, UK

${ }^{3}$ Department of Mathematics, Imperial College London, UK

${ }_{4}^{4}$ Psychedelic Research Group, Psychopharmacology Unit, Centre for Psychiatry, Imperial College London, UK

${ }^{5}$ Department of Psychology, Yale University, USA

${ }^{6}$ Mathematical Institute, University of Oxford, UK

${ }^{7}$ Center for Brain and Cognition, Universitat Pompeu Fabra, ES

8 Instituci Catalana de la Recerca i Estudis Avanats (ICREA), Universitat Pompeu Fabra, ES

${ }^{9}$ Centre for Music in the Brain, Department of Clinical Medicine, Aarhus University, DK

10 Institut d'Études Avancées de Paris, FR

11 Life and Health Sciences Research Institute (ICVS), School of Medicine, University of Minho, PT

\section{Abstract}

Growing evidence from the dynamical analysis of functional neuroimaging data suggests that brain function can be understood as the exploration of a fixed repertoire of metastable connectivity patterns ('functional brain networks'), which potentially underlie different mental processes.

The present study characterizes how the brain's dynamical exploration of resting-state networks is rapidly modulated by intravenous infusion of psilocybin, a tryptamine psychedelic found in "magic mushrooms". We employed a data-driven approach to characterize recurrent functional connectivity patterns by focusing on the leading eigenvector of BOLD phase coherence at single-TR resolution. Recurrent BOLD phase-locking patterns (PL states) were assessed and statistically compared between pre- and post-infusion of psilocybin in terms of their probability of occurrence and transition profiles. Results were validated using a placebo session.

Recurrent BOLD PL states revealed high spatial overlap with canonical resting-state networks. Notably, a PL state forming a frontoparietal subsystem was strongly destabilized after psilocybin injection, with a concomitant increase in the probability of occurrence of another PL state characterized by global BOLD phase coherence. These findings provide evidence of network-specific neuromodulation by psilocybin and represent one of the first attempts at bridging molecular pharmacodynamics and whole-brain network dynamics.

\section{Introduction}

Brain dynamics can be understood as the exploration of activity configurations over both space and time (Tononi and Edelman, 1998, Watanabe et al., 2014, Cabral et al., 2017b, Gu et al., 2018). This exploration may be defined in terms of trajectories within a fixed repertoire of 
metastable activity patterns, which potentially underlie different brain processes. Indeed, a robust repertoire of large-scale functional networks has been consistently detected across individuals and neuroimaging modalities, not only task performance but also during rest (Damoiseaux et al., 2006, De Luca et al., 2006, Mantini et al., 2007, Seeley et al., 2007, Musso et al., 2010, Brookes et al., 2011, Yeo et al., 2011, Hipp et al., 2012), indicating that brain function involves the coordinated integration of information over a repertoire of spatially distributed networks of specialized brain areas (Varela, 1979, Bressler and Menon, 2010, Menon, 2011, Cavanna et al., 2017, Lord et al., 2017). While the mechanisms driving the spontaneous formation and dissolution of functional networks remain under debate (Cabral et al., 2017a), brain function has recently been explored in terms of transitions between recurrent states of functional connectivity (Hansen et al., 2015, Cabral et al., 2017a, Cavanna et al., 2017, Tewarie et al., 2018). Although the functional relevance of these explorative dynamics during rest remains unclear (Christoff et al., 2016), recent evidence suggests that transitions between brain states are organized in a hierarchical manner (Vidaurre et al., 2017) and relate to cognitive function (Cabral et al., 2017b).

Novel insights into the neurobiological correlates of functional network states and their relationship to behaviour in both health and disease can be gained by understanding how specific psychoactive compounds modulate the relative stability of functional networks over time, as well as the transitions between them. Toward this aim, the present study investigates how psilocybin (4-phosphoryloxy-N,N-dimethyltryptamine) changes the exploration of the brain's dynamical repertoire. Psilocybin is a prodrug of psilocin (4-OH-N,N-dimethyltrayptamine) - a psychoactive ingredient found in so-called "magic mushrooms" and classical tryptamine 'psychedelic'. The subjective effects of psilocybin/psilocin include broadly unconstrained perception and cognition, hyper-associative cognition and, at higher doses, a breakdown in the perception of time, space and selfhood (Griffiths et al., 2006, Carhart-Harris et al., 2014). Psilocybin provides a promising experimental framework for linking molecular pharmacodynamics to changes in the brain's dynamical repertoire because its potent psychoactive effects are selectively due to its agonist activity at the serotonin $2 \mathrm{~A}\left(5-\mathrm{HT}_{2 \mathrm{~A}}\right)$ receptor (McKenna et al., 1990, Vollenweider et al., 1998, Passie et al., 2002, González-Maeso et al., 2007) - see Beliveau et al. (2017) for the highest resolution in vivo mapping of 5- $\mathrm{HT}_{2 \mathrm{~A}}$ receptor densities in the human brain. Furthermore, investigating how psilocybin modulates the exploration of functional network states over time may help better understand the functional mechanisms underlying the recently demonstrated therapeutic potential of psilocybin (and other indolealkylamines) for disorders including depression, anxiety and addiction (McKenna et al., 1990, Griffiths et al., 2006, Grob et al., 2011, Meltzer et al., 2012, Johnson et al., 2014, CarhartHarris et al., 2016).

The first fMRI investigation of psilocybin's effects on the human brain consisted of a taskfree paradigm in which healthy subjects were intravenously injected with the compound inside the scanner (or a placebo, at least 1 week apart) to characterize changes in brain activity under 
the influence of the drug (Carhart-Harris et al., 2012). The first dynamical analysis of this dataset found increased variance of intra-network synchrony over time for a number of canonical restingstate networks under psilocybin (Carhart-Harris et al., 2014). Somewhat consistently, a subsequent analysis of this same fMRI dataset using sliding-window correlations within a specific network of four brain regions (comprising the bilateral hippocampus and anterior cingulate cortex) revealed both a larger repertoire of functional motifs under psilocybin as well as greater entropy of the motif sequence (Tagliazucchi et al., 2014). Moreover, another experiment using MEG revealed increased Lempel-Ziv complexity (Lempel and Ziv, 1976), a measure of neural signal diversity, following psilocybin administration relative to placebo (Schartner et al., 2017). However, it has not yet been explored how psilocybin modulates the relative of specific functional network states over time.

Recently, a number of methodological approaches have been proposed to analyze BOLD connectivity dynamics at high temporal resolution (i.e., single volume / TR), focusing either on BOLD co-activation patterns (Tagliazucchi et al., 2012, Liu and Duyn, 2013, Karahanoğlu and Van De Ville, 2015), or on BOLD phase coherence patterns (Glerean et al., 2012, Hellyer et al., 2015, Cabral et al., 2017b). Whereas the first method considers a functional connection only when the BOLD signals are simultaneously increased, the latter is sensitive to the transient synchronization of BOLD signal fluctuations and does not distinguish between co-activations and codeactivations. In the light of recent evidence - supported by computational models - indicating that resting-state networks are the expression of the transient synchronization of different functional subsystems, we predict that the methods relying on the BOLD signal phase rather than on its amplitude will be more sensitive to capture this ultra-slow oscillatory dynamics governing the formation of functional networks (Deco et al., 2009, Cabral et al., 2014a, Ponce-Alvarez et al., 2015, Deco and Kringelbach, 2016, Cabral et al., 2017a, Gutierrez-Barragan et al., 2018).

To identify recurrent patterns of BOLD phase coherence across subjects and quantify differences in the exploration of the repertoire of functional networks in the current dataset, we employed a recently developed data-driven approach, the Leading Eigenvector Dynamics Analysis (LEiDA), which captures instantaneous phase-locking patterns with reduced dimensionality by considering only the relative phase of BOLD signals (i.e., how all BOLD phases project into their leading eigenvector at each discrete time point) (Cabral et al., 2017b, Figueroa et al., 2019). LEiDA appears as a valuable step in the search for functional network recurrences in dynamical analysis because the reduced dimensionality (from a NxN matrix to a $1 \times N$ vector) allows for better convergence of the clustering algorithm, revealing robust BOLD phase-locking patterns that consistently reoccur across fMRI scans for different subjects. However, despite returning meaningful functional susbsystems in previous studies (Cabral et al., 2017b, Figueroa et al., 2019), the recurrent patterns identified by LEiDA have not yet been qualitatively compared to canonical resting-state networks (Damoiseaux et al., 2006, Yeo et al., 2011).

In the current work, we therefore employed the LEiDA approach to identify recurrent BOLD phase-locking patterns (PL states) and quantified differences in their probability of 
occurrence and transition profiles before and after the infusion of psilocybin, while subjects were inside the MRI scanner. The validity of the results was subsequently verified using the placebo dataset, and the repertoire of PL patterns returned by LEiDA was compared to well-established canonical resting-state networks.

\section{Methods}

The dataset used in the present study has been previously published. A brief description of the participants, protocol and acquisition is included below. Please see Carhart-Harris et al., 2012 for further information.

\section{Participants}

Employing rigorous standards for data quality, namely minimal motion during the scan, at least 21 years of age, no personal or family history of a major psychiatric disorder, no substance dependence, no cardiovascular disease, and no history of adverse response to a psychedelic drug yielded datasets for nine participants. All subjects had used psilocybin at least once before, but not within 6 weeks of the study. All participants gave informed consent to participate in the study. The study was approved by a National Health Service research ethics committee.

\section{Experimental protocol}

All subjects underwent two 12 minute eyes-closed resting state $\mathrm{fMRI}$ scans over separate sessions, at least 7 days apart. In each session, subjects were injected intravenously with either psilocybin ( $2 \mathrm{mg}$ dissolved in $10 \mathrm{ml}$ saline, 60 seconds intravenous injection) or a placebo (10 ml saline, 60 seconds intravenous injection) in a counterbalanced design (see Figure 2 for an illustration of the scanning paradigm). Injections were given manually by a doctor within the scanning suite. The infusions began exactly $6 \mathrm{~min}$ after the start of the $12 \mathrm{~min}$ scans and lasted 60 seconds. The subjective effects of psilocybin were felt almost immediately after injection and sustained for the remainder of the scanning session. This experimental approach thus provided four distinct $\mathrm{fMRI}$ recordings (6 minutes each) corresponding to each of four experimental conditions: pre/post placebo injection and pre/post psilocybin injection. To account for potential confounds related to the infusion event and to focus analyses on the active drug state, the last 5 min of each scan were used for the relevant analyses. In order to have BOLD timeseries of equal length pre- and post-injection, only the first 5 minutes of scanning (pre-infusion) were considered in the analysis. At the end of each session, subjects were asked to rate the overall intensity of their subjective experience under the drug (or placebo) and to comment on their wakefulness level throughout the scan. As expected, all participants rated the subjective effects of psilocybin (mean intensity $=6.9 / 10 \pm 2.6$ ) as much stronger than placebo (mean intensity $=0.4 / 10 \pm 0.6$ ) and none of the subjects reported falling asleep during either scanning session. 


\section{Neuroimaging data acquisition \& processing}

\section{Anatomical scan acquisition}

Neuroimaging data were acquired using a 3T GE HDx MRI system. Anatomical scans were performed before each functional scan and thus prior to administering either the drug or placebo. Structural scans were collected using a 3D fast spoiled gradient echo scans in an axial orientation, with field of view $=256 \times 256 \times 192$ and matrix $=256 \times 256 \times 192$ to yield $1 \mathrm{~mm}$ isotropic voxel resolution (repetition time/echo time TR/TE $=7.9 / 3.0 \mathrm{~ms}$; inversion time $=450 \mathrm{~ms}$; flip angle $=20$ ).

\section{fMRI acquisition}

BOLD-weighted $\mathrm{fMRI}$ data were acquired using a gradient echo planar imaging sequence, TR/TE 3000/35 ms, field-of-view $=192 \mathrm{~mm}, 64 \times 64$ acquisition matrix, parallel acceleration factor $=2$, 90 flip angle. Fifty-three oblique axial slices were acquired in an interleaved fashion, each $3 \mathrm{~mm}$ thick with zero slice gap ( $3 \times 3 \times 3 \mathrm{~mm}$ voxels).

\section{fMRI processing}

fMRI data were processed using MELODIC (Multivariate Exploratory Linear Decomposition into Independent Components) (Beckmann and Smith, 2004), part of FSL (FMRIB's Software Library, www.fmrib.ox.ac.uk/fsl). The default parameters of this imaging pre-processing pipeline were used on all participants: motion correction using MCFLIRT (Jenkinson et al., 2002), non-brain removal using BET (Smith, 2002), spatial smoothing using a Gaussian kernel of FWHM 5mm; grand-mean intensity normalization of the entire 4D dataset by a single multiplicative factor and linear de-trending over 50 second intervals.

We used the Anatomical Automatic Labeling (AAL) atlas (Tzourio-Mazoyer et al., 2002) to parcellate the $\mathrm{MNI}$ brain into $N=90$ cortical and sub-cortical non-cerebellar brain areas and the BOLD signals were then averaged over all voxels belonging to each brain area using FSL tools. The BOLD signals in each of the 90 brain areas were subsequently band-pass filtered between 0.02 and $0.1 \mathrm{~Hz}$ (using a $2^{\text {nd }}$ order Butterworth filter), discarding in this way the high frequency components associated to cardiac and respiratory signals $(>0.1 \mathrm{~Hz})$, and focusing on the most meaningful frequency range of resting-state fluctuations (Biswal et al., 1995).

For each subject, this procedure was applied separately to the fMRI data from all four experimental conditions listed above, resulting in four $N x T$ BOLD datasets where $N=90$ is the number of brain areas and $T=100$ is the number of TRs.

\section{Dynamic BOLD phase-locking analysis}

To compute the phase alignment between each pair of AAL regions, first the BOLD phases, $\theta(n, t)$, were estimated using the Hilbert transform for each BOLD regional timecourse (see Figure $1 \mathrm{~A}$ ) (Glerean et al., 2012, Cabral et al., 2017b). The Hilbert transform expresses a given signal $x$ as $x(t)$ $=A(t) * \cos (\theta(t))$, where $A$ is the time-varying amplitude and $\theta$ is the time-varying phase. In Figure $1 A$, we represent the $B O L D$ signal phase of one area $n$ over time as $e^{i \theta(t)}$ with $\sin (\theta(t))$ representing 
the imaginary part of the analytic phase, and $\cos (\theta(\mathrm{t}))$ representing its real part (black dotted lines). We show that $\cos (\theta(t))$ captures the oscillatory dynamics of the original BOLD signal (green) - which is mostly preserved after band-pass filtering between 0.02 and $0.1 \mathrm{~Hz}$ (blue) - but with constant amplitude (i.e., between -1 and 1 ). The arrows in red represent the Hilbert phases at each TR, which can be projected into the complex plane (i.e., the plane defined by the real and imaginary axes, represented at $\mathrm{t}=0$ ).

Figure $1 \mathrm{~B}$ shows, at a single time point $\mathrm{t}$, all $\mathrm{N}=90 \mathrm{BOLD}$ phases in the cortical space, placed at the center of gravity of each brain area. Here, the arrows are colored according to their direction when projected into the leading eigenvector of phase coherence. On the middle of panel $\mathrm{B}$, the same $\mathrm{N}=90$ BOLD phases are plotted in the complex plane, i.e., all centered at the same origin.

To obtain a whole-brain pattern of BOLD phase coherence at each single time point $t$, we compute a dynamic Phase-Locking matrix $\mathrm{dPL}(n, p, t)$ which estimates the phase alignment between each pair of brain areas $n$ and $p$ at each time $t$ using Equation (1):

$\operatorname{dPL}(n, p, t)=\cos (\theta(n, t)-\theta(p, t))$.

Using the cosine function, two areas $n$ and $p$ with temporarily aligned BOLD signals (i.e., with no phase difference) at a given TR will have a phase-locking value $d P L(n, p, t)=\cos \left(0^{\circ}\right)=1$. On the other hand, time points when the BOLD signals have $180^{\circ}$ phase difference (in complex plane) will have $d P L(n, p, t)=\cos \left(180^{\circ}\right)=-1$. The cosine function being even, the $d P L(t)$ matrix is symmetric with values ranging between -1 and 1 .

The resulting $d P L$ for each subject in each condition is thus a three-dimensional tensor with size $N x N x T$, where $N=90$ is the number of brain areas and $T=100$ is the total number of time points. 

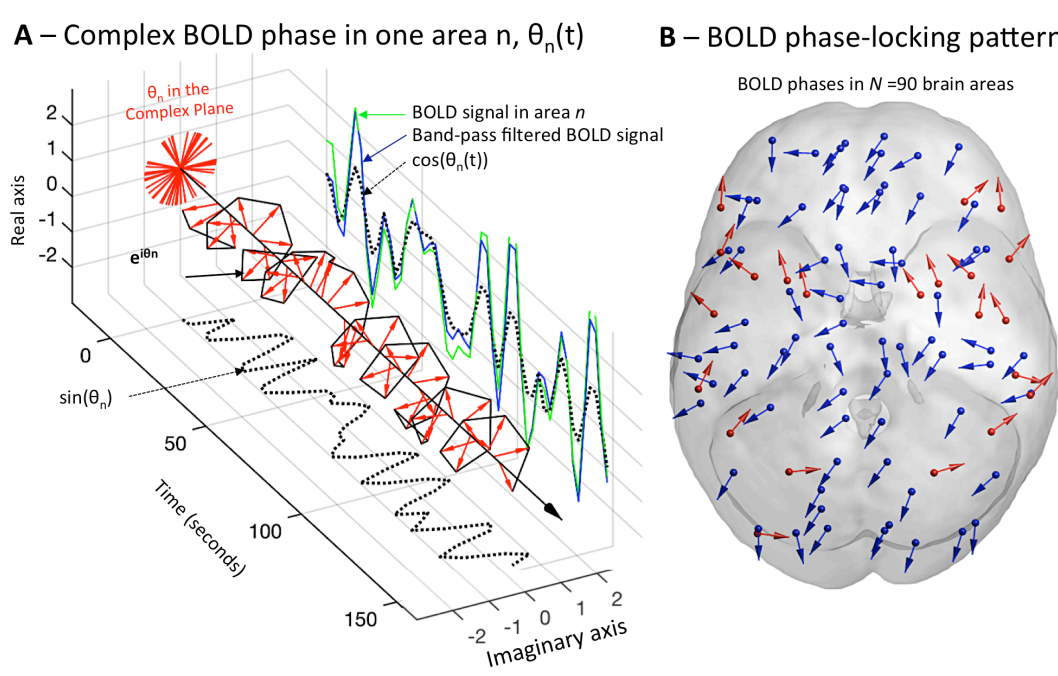

\section{C- Detecting recurrent BOLD phase-locking patterns}
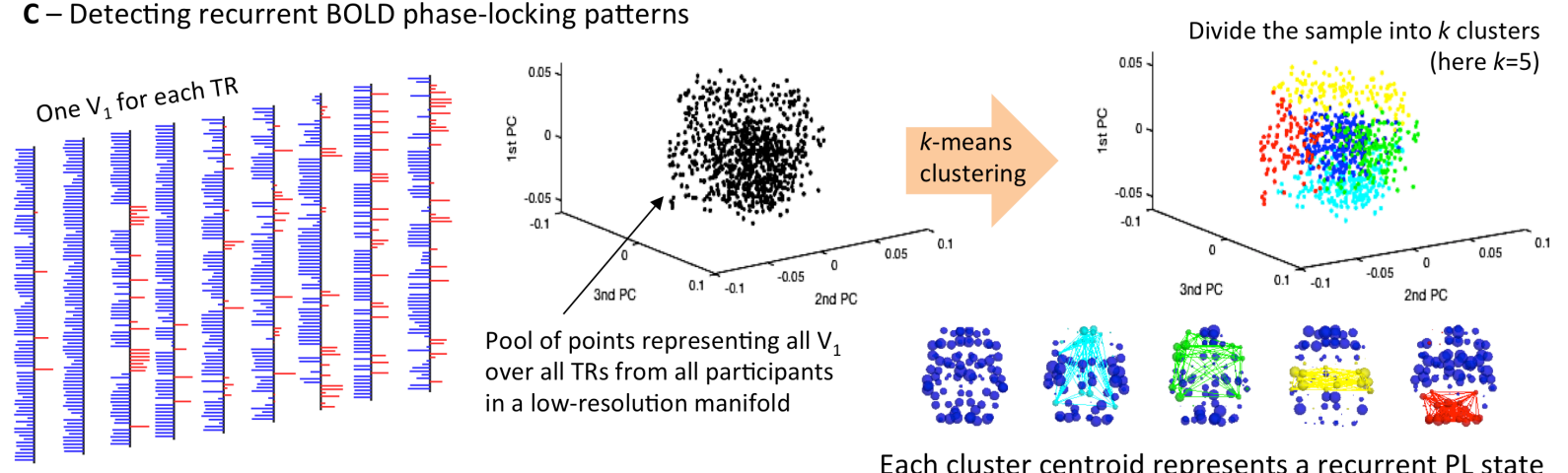

Pool of points representing all $V_{1}$ over all TRs from all participants in a low-resolution manifold
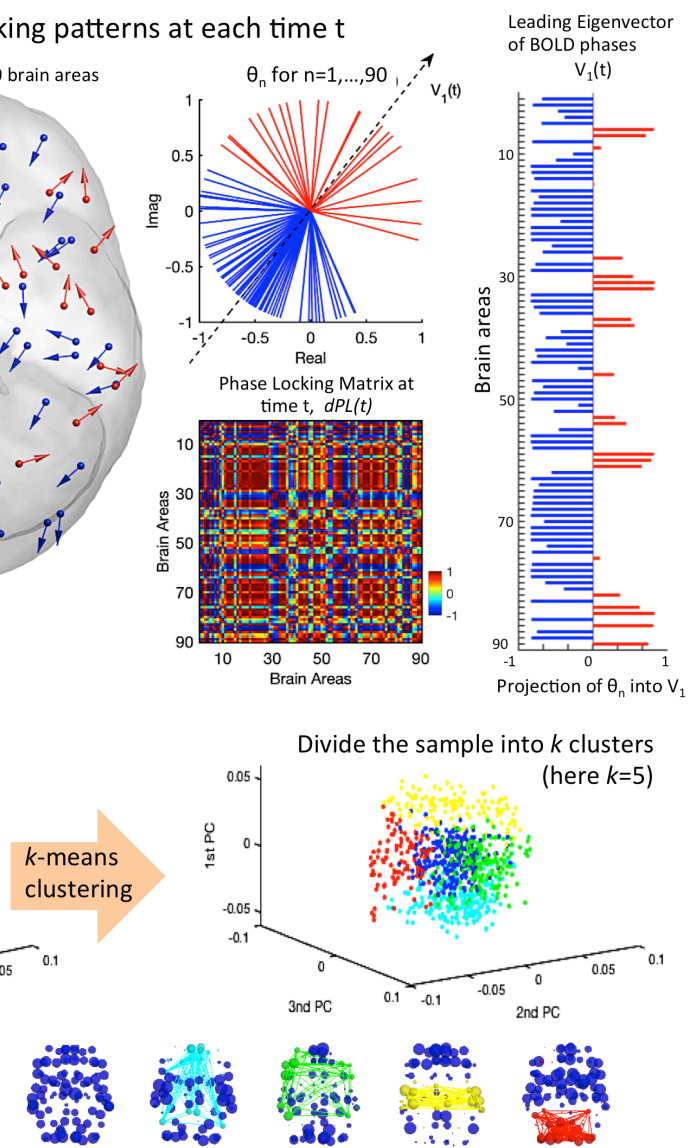

Each cluster centroid represents a recurrent PL state

Figure 1 - Detection of recurrent BOLD phase-locking (PL) patterns. A) The BOLD signal in a given brain area $\mathrm{n}$ (green) is first band-pass filtered between 0.02 and $0.1 \mathrm{~Hz}$ (blue) and then transformed into an analytic signal (with real and imaginary components) using the Hilbert transform. The phase dynamics of the analytic signal can be represented over time by $\mathrm{e}^{\mathrm{i} \theta}$ (black line), where the real part is captured by $\cos (\theta)$ and the imaginary part by $\sin (\theta)$ (black dotted lines). The red arrows represent the BOLD phases at each TR. B) At a single time point, the BOLD phases in all $\mathrm{N}=90$ brain areas are represented both in the cortical space (arrows centered at the center of gravity of each brain area) and in the complex plane (i.e., the unit circle with real and imaginary axes, where all phases are centered at the same origin). The phaselocking matrix at time $t$ captures the BOLD phase alignment between each pair of brain areas. (Right) The leading eigenvector of the phase-locking matrix at time $t, V_{1}(t)$, is the vector that best captures the main orientation of all BOLD phases (represented as a black dashed arrow in the unit circle in the left), where each element in $V_{1}(t)$ corresponds to the projection of the BOLD phase in each area into $V_{1}(t)$. The elements of $V_{1}(t)$ are colored according to their relative direction with respect to $V_{1}$ (red: positive; blue: negative). C) We take the leading eigenvector $V_{1}(t)$ as a low-dimensional representation of the BOLD phase-locking patterns over time. To identify recurrent phase-locking patterns, we apply a clustering algorithm (k-means), which divides the pool of data points into a predefined number of clusters k. Each cluster is represented by a central vector, which we take to represent a recurrent BOLD phase-locking pattern, or PL state. 


\section{Leading eigenvector of the phase-locking matrix}

To characterize the evolution of the $\mathrm{dPL}$ matrix over time with reduced dimensionality, we employed a method termed Leading Eigenvector Dynamics Analysis (LEiDA) (Cabral et al., 2017b, Figueroa et al., 2019). The leading eigenvector of the NxN phase-locking matrix at time $t, V_{1}(t)$, is a $\mathrm{N} \times 1$ vector that captures the main orientation of BOLD phases over all areas, where each element in $\mathrm{V}_{1}(\mathrm{t})$ represents the projection of the BOLD phase in each brain area into the leading eigenvector (Figure $1 B$, right). When all elements of $V_{1}(t)$ have the same sign, all BOLD phases are pointing in the same direction (half-plane) with respect to the orientation determined by $V_{1}(t)$, which is indicative of a global mode governing all BOLD signals. If instead the first eigenvector $\mathrm{V}_{1}(\mathrm{t})$ has elements of different signs (i.e., positive and negative), the BOLD signals follow different directions with respect to the leading eigenvector, which naturally divides the brain areas into 2 clusters according to their BOLD phase relationship (see Figure 1B). Moreover, the magnitude of each element in $V_{1}(t)$ indicates the 'strength' with which brain areas belong to the communities in which they are placed (see Newman (2006) and the Supplementary Information for further details). Since $V$ and $-V$ span the same one-dimensional subspace, we use a convention ensuring that most of the elements have negative values, because, as we will show, our results revealed that the smallest group of areas whose BOLD phases do not follow the global mode reveal meaningful functional brain networks (i.e., canonical resting-state networks).

This approach substantially reduces the dimensionality of the data, while still explaining most of the variance of BOLD phase coherence (see the Supplementary Figure S1 where we show that the leading eigenvector consistently represents $>50 \%$ of the variance in phase coherence at all time points).

\section{BOLD phase-locking states}

\section{Detection of recurrent BOLD phase-locking patterns}

To identify recurrent PL patterns, we applied a k-means clustering algorithm to divide the set of 1800 eigenvectors (corresponding to all 100 TRs of all 9 subjects both before and after psilocybin injection) into a predefined number of clusters $k$ (see Figure $1 C$ ), with higher $k$ revealing more rare and more fine-grained patterns. Since the optimal number of functional networks to consider remains an open question, we ran the k-means clustering algorithm with $k$ ranging from 5 to 10 (i.e., dividing the set of eigenvectors into $k=5,6, \ldots, 10$ clusters) to cover the range of functional networks commonly reported in the resting-state fMRI literature (Beckmann et al., 2005, Damoiseaux et al., 2006, Yeo et al., 2011).

For each partition model considered (i.e., $k=5$ to $k=10$ ), the clustering returns $k$ cluster centroids in the shape of $\mathrm{Nx} 1$ vectors $\mathrm{V}_{\mathrm{C}}$, which represent the average vector of each cluster (in Figure $2 \mathrm{~A}$ we show the 7 central vectors obtained with $k=7$; the centroids obtained for all solutions ranging from $k=5$ to $k=10$ are shown in Supplementary Figure S2). We take these central vectors as representing recurrent BOLD phase-locking states, or PL states. As shown in Figure $2 \mathrm{~B}$, each $\mathrm{PL}$ state can be represented as a network in cortical space, where the value of $V_{c}(n)$ is used to scale 
the color of each brain area and links are plotted between areas with positive sign to highlight the network detaching from the global mode (in Figure 2 we used $V_{c}(n)>0.3$ for visualization purposes only). Also, to facilitate visualization and interpretation of PL states, the cluster centroid vectors $\mathrm{V}_{C}$ can be rendered onto a cortical surface, e.g. using the HCP Workbench (Figure 2C). Finally, we note that the PL states can also be represented back into matrix format (NxN) by computing the outer product $\mathrm{V}_{\mathrm{C}} . \mathrm{V}_{\mathrm{C}}^{\top}$ resulting in a matrix of rank 1 (i.e., a matrix that is obtained from a single vector), with positive values between all elements with the same sign in $\mathrm{V}_{\mathrm{C}}$ (be they positive or negative), and negative values between elements of different signs (Figure 2D). 


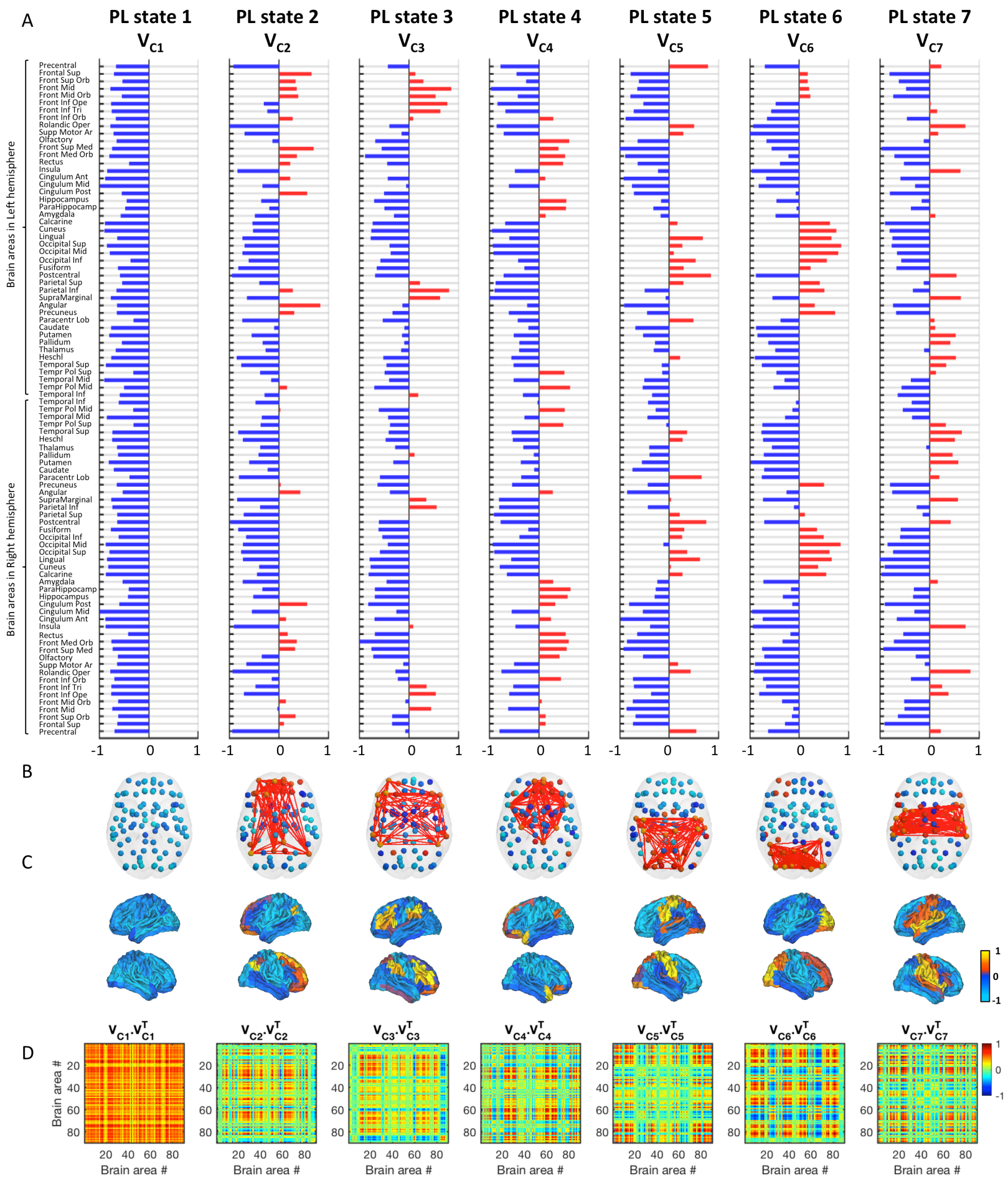

Figure 2 - Repertoire of recurrent BOLD phase-locking patterns (PL states) obtained by clustering the leading eigenvectors of BOLD phase coherence (here with $k=7$ ). Seven recurrent PL states were obtained from unsupervised clustering of the 1800 eigenvectors of BOLD phase coherence over time, sorted (left to right) according to decreasing probability of occurrence before psilocybin injection. Each PL state is represented by the central vector of each cluster $V_{C}$ as: $A$ ) a bar plot showing the $N$ elements in $V_{C}$, 
representing the projection of the BOLD phase in each brain area into the leading eigenvector; B) a network in cortical space, where the value of $V_{c}(n)$ is used to scale the color of each brain area and links are plotted between areas with $V_{c}(n)>0.3$ to highlight the network detaching from the global mode; $C$ ) cortical renderings in AAL space of regional contributions to the respective $V_{C}$ and $D$ ) a matrix obtained by calculating the outer product of $V_{C}$, where positive values are the product of $V_{C}$ elements with the same sign, be they positive or negative.

\section{Probability of occurrence and switching profiles of PL states}

The clustering assigns to each TR a single PL state, by selecting the closest centroid $V_{c}$ at each TR, as illustrated by the color-coded bars in Figure 3A for one representative subject (the color-coded cluster time courses are shown for all 9 subjects in Supplementary Figures S3 and S4) Using the state time courses, we calculated the probability of occurrence of each state, which is simply the number of epochs assigned to a given PL state divided by the total number of epochs (TRs) in each scanning session (see Figure $3 \mathrm{~A}$ for an illustration with $\mathrm{k}=7$ ). For each partition model (i.e., with $k=5$ to $k=10$ ) the probabilities of each PL state were calculated for each subject before and after psilocybin injection separately.

Differences in probabilities of occurrence before and after injection were statistically assessed using a permutation-based paired t-test. This non-parametric test uses permutations of group labels to estimate the null distribution, which is computed independently for each experimental condition (before versus after psilocybin injection). For each of 1000 permutations, a t-test is applied to compare populations and a $p$-value is returned.

A

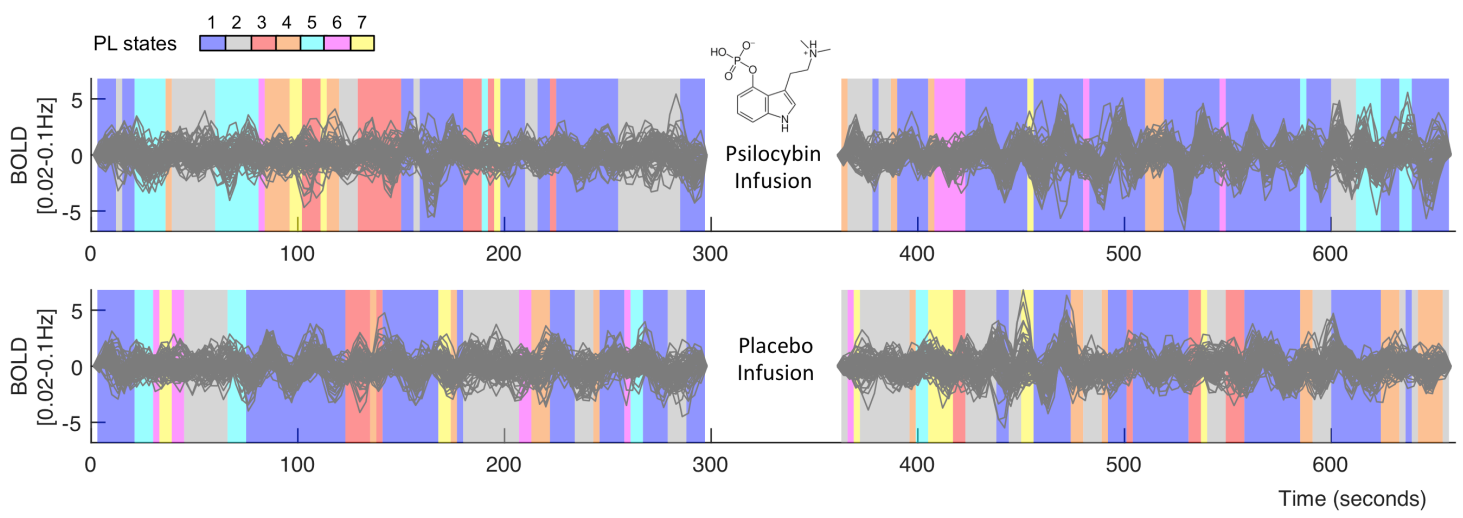

B

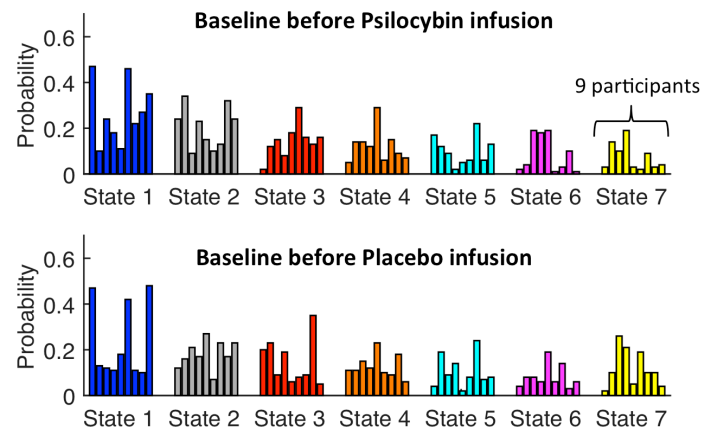

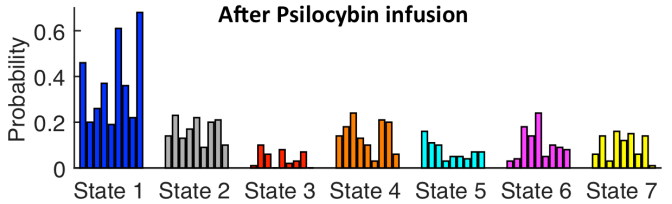

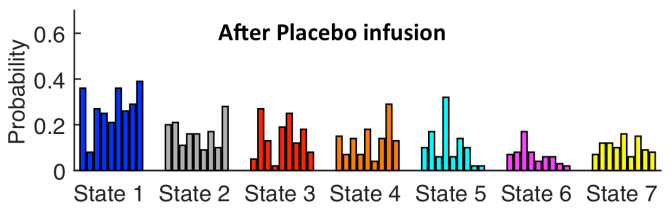


Figure 3 - Assignment of one BOLD Phase-Locking (PL) state to each TR and corresponding probabilities of occurrence for each condition and participant. A) For each of the 9 participants, $2 \mathrm{fMRI}$ sessions were recorded at least one week apart. In the middle of each session, either Psilocybin or a Placebo was intravenously administered inside the scanner. The color-coded bars behind the BOLD signals represent the PL state assigned to each TR (corresponding to the 7 PL states reported in Figure 2). Results are shown here for subject 9 (the remaining participants are shown in SI Figures S3 and S4). B) The probabilities of occurrence of each PL state were obtained for each of the 4 conditions and for each subject separately, which were used for the subsequent statistical analysis.

\section{Selection of the optimal number of PL states}

In the current study we did not aim to determine the optimal number of PL states governing resting-state activity, but rather to investigate if there are PL state(s) that significantly differ in their probability of occurrence following the administration of psilocybin. As such, we employed a data mining approach to detect meaningful activity patterns (PL states) from the session during which psilocybin was infused, and left the placebo session (recorded more than 7 days apart) as a validation dataset (see section Validation using the Placebo dataset).

To search for PL patterns affected by psilocybin, we varied $k$ (number of clusters) between 5 and 10 , and for each $k$ examined how the probability of occurrence of of each PL state changed after the injection of psilocybin. We subsequently analyzed the the robustness of the results over the range of partition models by plotting the $\mathrm{p}$-values with respect to different significance thresholds, i.e., the standard $\alpha_{1}=0.05$ or $\alpha_{2}=0.05 / \mathrm{k}$ to correct for the number of independent hypothesis compared in each partition model.

For the subsequent validation using the placebo condition, we used exactly the same PL states obtained previously for the psilocybin session for the selected optimal $k$, and evaluated how these same PL states were explored during the placebo session. As in the previous analysis, we first obtained the 1800 leading eigenvectors $V_{1}$ ( 9 subjects, 2 conditions, 100 TRs each) and ran a single iteration of the $k$-means algorithm with $k=7$, defining as 'start vectors' the 7 cluster centroids $V_{c}$ obtained before. This computes the cosine distance between each observation in the placebo dataset and the previously defined 7 cluster centroids, returning a sequence of cluster time courses for the placebo fMRI session. Critically, this approach allows a direct comparison with the psilocybin fMRI sessions recorded several days apart, and enabling us to verify whether the probabilities of approaching a given cluster centroid in the placebo sessions were: i) consistent with the pre-psilocybin condition and ii) different from the post-psilocybin condition.

In a follow-up analysis, we compared the number of within-subject occurrences of the most meaningful PL states before and after the psilocybin injection using a paired t-test.

\section{Comparison with resting-state networks}

We first transformed the 7 RSNs defined in $2 \mathrm{~mm}^{3} \mathrm{MNI}$ space by (Yeo et al., 2011) into 7 vectors with 90 elements each (shown in Figure 4), where each element scales the contribution of each 
AAL brain area to the corresponding RSN. Subsequently, we computed the bivariate correlation with the centroid vectors $V_{C}$, setting all negative elements in $V_{C}$ to zero, keeping only the values of the positive elements (the red links in Figure 2B).

\section{Global order and metastability}

Using a perspective from dynamical systems theory, we investigated how psilocybin affected the order and stability of BOLD signals. At each instant of time, the degree of order between BOLD phases $\theta(n, t), \mathrm{n}=1, . ., 90$, can be quantified using the magnitude of the Kuramoto Order Parameter, $O P(t)$, which can range between 0 (when all BOLD signals are out of phase) and 1 (when all BOLD signals are in phase):

$O P(t)=\frac{1}{N}\left|\sum_{n=1}^{N} e^{i \theta(n, t)}\right|$.

The mean magnitude of the Order Parameter over time informs whether the system is mostly incoherent, partially synchronized or fully synchronized (Acebron et al., 2001, Acebrón et al., 2005, Cabral et al., 2011, Deco and Kringelbach, 2016). Moreover, the standard deviation of the order parameter, STD(OP), can be used to characterize the degree of metastability in the system. In other words if the order parameter is constant over time, it means that the system is in a stable equilibrium, be it synchronized or not. When more than one weakly stable states co-exist and the system systematically switches from one to another, then the variance of the order parameter increases, indicating a dynamical system with metastability (Shanahan, 2010, Cabral et al., 2014b). To investigate the effects of psilocybin on the stability of the global order of BOLD phases, we therefore calculated the standard deviation of the order parameter over time within each subject before and after the psilocybin infusion. Between-condition differences on these measures were assessed using a one-sample t-test.

\section{Results}

\section{Repertoire of PL states reveals canonical resting-state networks}

The repertoire of PL states obtained when dividing the set of leading eigenvectors into seven clusters is plotted in Figure 2, where each state is represented by its central vector $V_{c}$ as: $(A)$ a bar plot showing the projection of the BOLD phase in each brain area into the leading eigenvector, (B) a network in cortical space, where links are plotted between areas with $V_{C}(n)>0.3$ to highlight the network detaching from the global mode; $(C)$ a rendering of regional values of $V_{C}$ onto the cortical surface, and (D) a matrix obtained by calculating the outer product of $V_{c}$. While partitions with different $k$ were produced, we selected the partition into $k=7$ PL states because it returned the PL state that most significantly differed in terms of probability of occurrence after psilocybin injection, as we will explain in section Results - Consistency of between-condition differences across partition models.

Results reveal that the most probable BOLD phase-locking state (PL state 1 ) corresponds to a state where all BOLD signals are following one main direction (i.e., all projecting toward the same direction into the leading eigenvector), in line with findings from prior studies using LEiDA (Cabral 
et al., 2017b, Figueroa et al., 2019). While this global modulation occurs for significant a fraction of the time, we detect a number of non-global patterns that transiently and recurrently shape the phase alignment of BOLD signals during the remaining time.

The non-global PL states 2-7 shown in Figure 3 reveal striking spatial similarities with canonical resting-state networks reported in the literature. We verified this overlap by computing the correlation between the 7 resting-state networks (RSNs) defined in Yeo et al. (2011) and each PL state obtained herein (Figure 4). Corroborating our observations, we found that all non-global PL states show strong and statistically significant spatial overlap with well-documented resting-state networks, namely the Default Mode network (PL state 2; Pearson's $r=0.35, p=5.5^{*} 10^{-4}$ ), the Frontoparietal network (PL state $3 ; r=0.70, p=1.1 * 10^{-14}$ ), the Limbic network ( $P L$ state 4; $r=$ $\left.0.36, p=5.5^{*} 10^{-4}\right)$, the Somatomotor network ( $P$ L state $5 ; c c=0.65, p=6.1 * 10^{-12}$ ), and the Visual network ( $\mathrm{PL}$ state $6 ; \mathrm{r}=0.79, \mathrm{p}=2.3^{*} 10^{-20}$ ). Finally, $\mathrm{PL}$ state 7 reveals approximately equal contributions of the Ventral Attention $\left(r=0.43, p=2.0^{*} 10^{-5}\right)$ and the Somatomotor networks $(r$ $\left.=0.43, p=2.8 * 10^{-5}\right)$. 

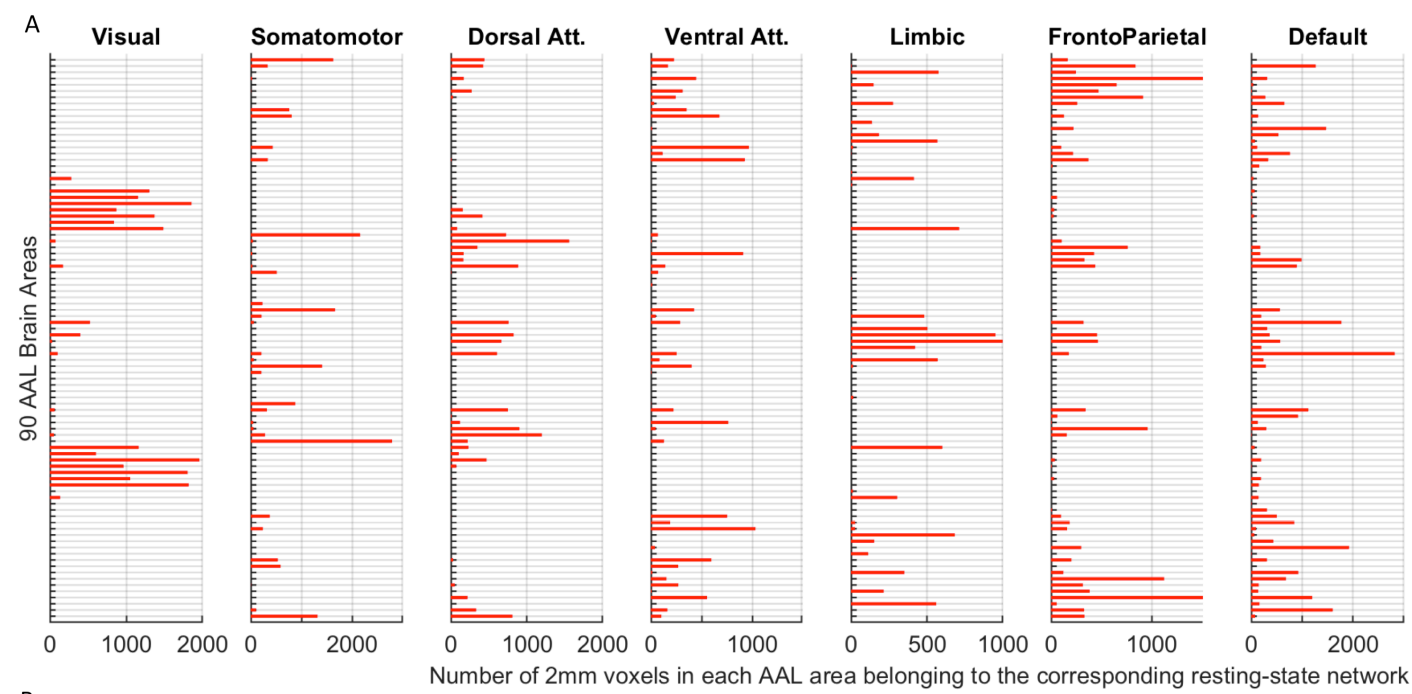

B

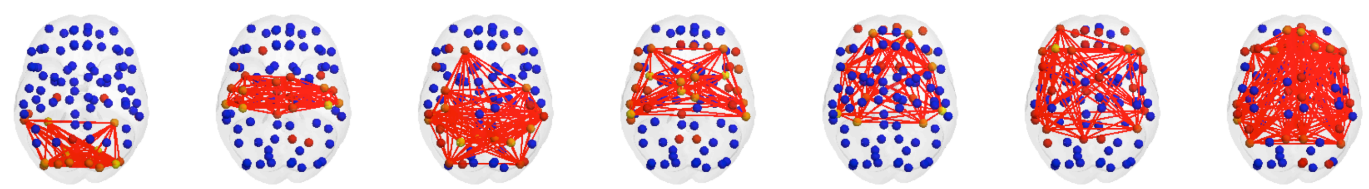

C

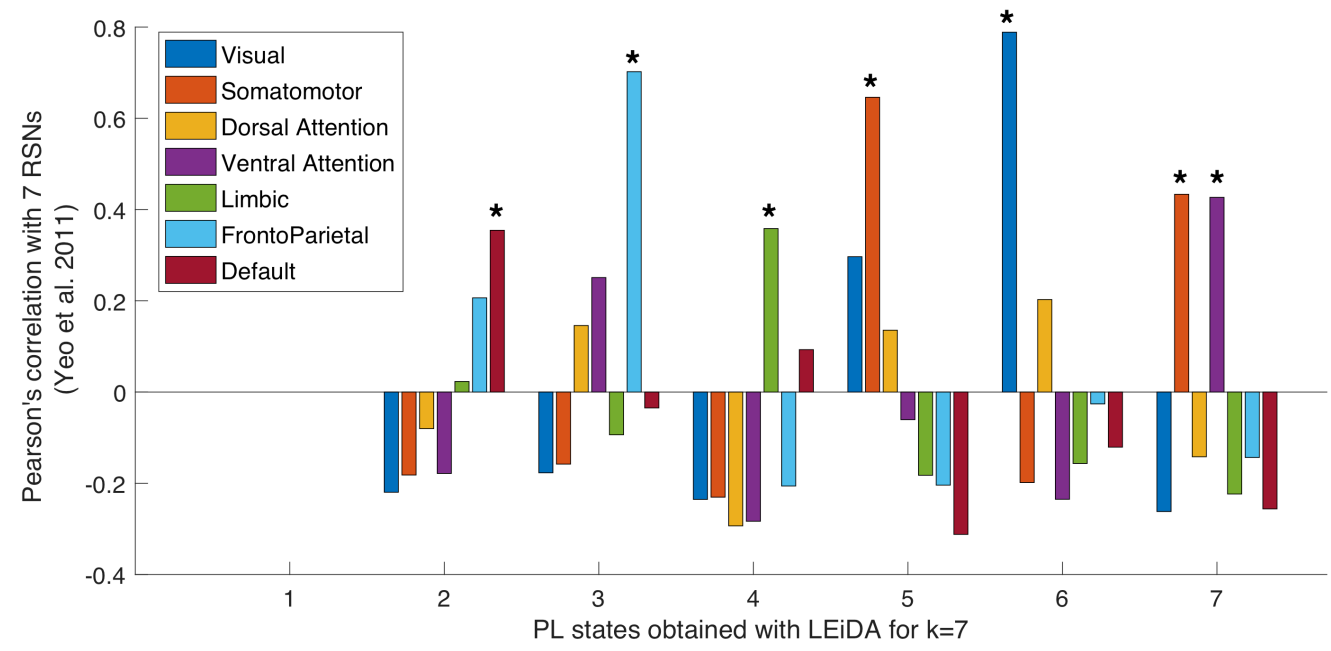

Figure 4 - Overlap of the 6 non-global PL states with seven canonical resting-state networks (RNSs). A The 7 cortical networks defined in Yeo et al. (2011) were transformed into AAL space by counting the number of $2 \mathrm{~mm}^{3} \mathrm{MNI}$ voxels in each AAL brain area that belong to each of the 7 networks, obtaining in this way a vector with $\mathrm{N}=90$ elements that we can compare with the PL states obtained in this work. B Representation in cortical space of the vectors shown in (A) colouring in red the spheres representing the brain areas contributing to each RSN. Red links were plotted between AAL areas with $>400 \mathrm{MNI}$ voxels contributing to each RSN. C - We compared the 6 non-global PL states obtained with LEiDA (for $k=7$ ) with the 7 RSNs by computing the Pearson's correlation between the $1 \times 90$ centroid vectors $V_{c}$ (shown in Figure 3 ) and the vectors shown in (A). Asterisks denote significant correlations with $p$-value $<0.001$. 


\section{Consistency of between-condition differences across partition models}

The repertoire of PL states obtained depends on the number of clusters $k$ defined in the $k$-means clustering algorithm, with a higher $k$ generally revealing more fine-grained, less frequent and often less symmetric networks. Here, we chose to cover a range of partition models (i.e., with $k$ varying gradually between $k=5$ to $k=10$ ) and examined the $\mathrm{PL}$ configurations that most significantly differentiate brain activity under psilocybin in each partition model relative to the pre-injection baseline.

In Figure 5, we show, for each partition into $k$ PL states (horizontal axis), the $p$-values obtained from between-condition comparisons (pre vs post-psilocybin) in terms of probability of occurrence of each of the corresponding $k \mathrm{PL}$ states. Since a higher number of comparisons increases the chances of false positives (i.e., of $p$-values falling below the standard threshold of $\alpha_{1}=0.05$, shown in red in Figure $5 C$ ), we correct the threshold by the number of independent hypothesis tested within each partition model, i.e., $\alpha_{2}=0.05 / k$ (green dashed line in Figure 5C). Note that across partition models, the null hypotheses being tested are not independent from each other (as shown in the correlations reported in Figure 5D), so correcting for the full number of comparisons would not be adequate here.

We find that, for the entire range of partition models explored (i.e., $5 \leq k \leq 10$ ), the PL state that most strongly alters its probability of occurrence after the psilocybin infusion is a frontoparietal network (Figure 5A) passing the corrected threshold $\alpha_{2}$ for all $k$ except for $k=6(p=0.0095)$, which despite falling below $\alpha_{1}$, narrowly misses the $\alpha_{2}$ cutoff. The most significantly different PL state of each partition model is reported in Figure 5B in vector format. These PL states were highly correlated across clustering solutions ( $C C>0.86$ for all pairs of PL states, Figure 5D), which indicates that they refer to the same underlying functional network, with small differences arising from the number of output states constrained by $k$. Furthermore, we note that a second network exhibited significant differences between conditions surviving the correction for the number of clusters $\left(\alpha_{2}=0.05 / k\right)$ for the clustering solutions: $k=5(p=0.0078)$ and $k=7(p=0.0068)$ (Figure $5 \mathrm{C}$, second smallest $\mathrm{p}$-value for each $\mathrm{k}$ ). This network corresponds to the globally coherent $\mathrm{PL}$ state 1, which also showed a high level of consistency across the range of clustering solutions, with some brain areas being more strongly aligned to the global mode of BOLD phase coherence than others (see Supplementary Figure S2). 
A

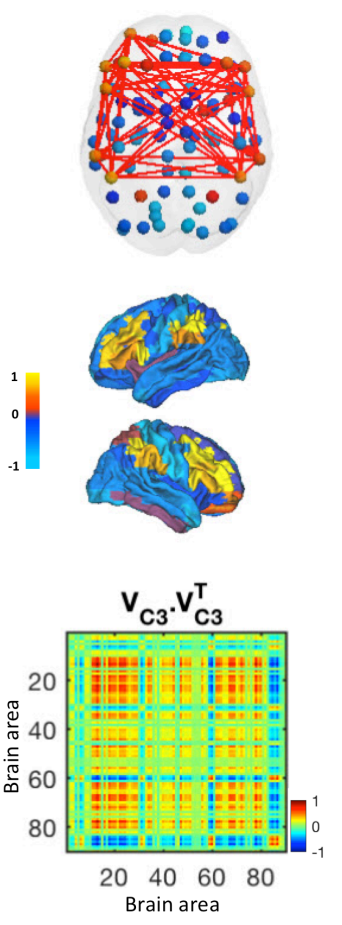

B

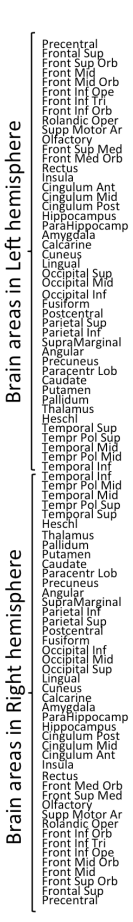

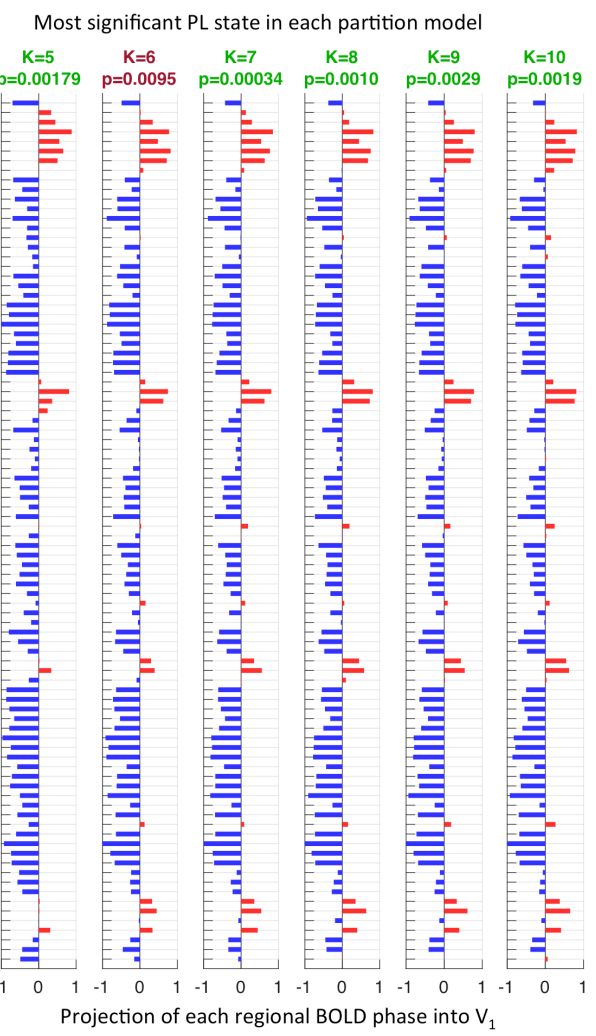

C Statistical significance of PL states between conditions

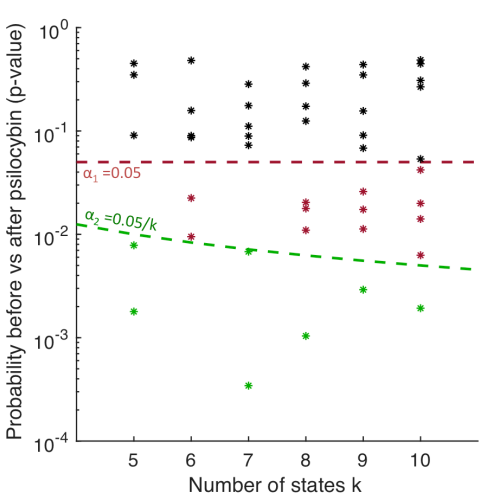

D

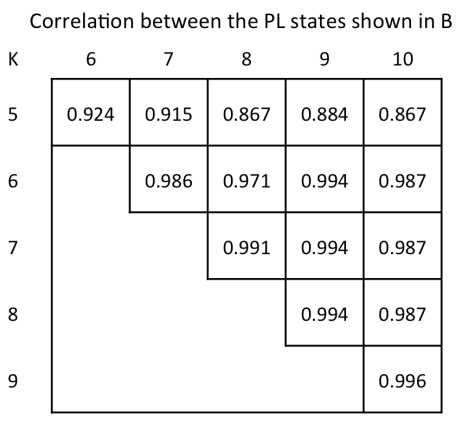

Figure 5 - Detection of the PL states that most significantly change their probabilities of occurrence after psilocybin infusion across partition models. A) The PL state differing the most between conditions is a functional network consisting of lateral frontoparietal areas, shown here for $k=7$ (PL state 3 from Figure 2). B) Bar plots showing, for each partition into $k=5$ up to $k=10$ PL states, the cluster centroid of the PL state that most significantly changed in probability of occurrence between conditions ( $p$-values reported in the title are colored according to significance level). C) For each partition model, we plot the p-values associated to the comparison between all PL state probabilities before and after psilocybin injection. We find that, although most PL states do not show significant differences between groups (black dots falling above the $\alpha_{1} \quad 0.05$ threshold), some PL states show significant differences in probability of occurrence between groups, falling below (or very near for $k=6$ ) the corrected threshold by the number of clusters ( $\alpha_{2}=0.05 / \mathrm{k}$, green dashed line). D) The PL states representing the Frontoparietal network in different partition models (shown in B) are highly correlated with one another.

\section{Network-specific neuromodulation}

The probabilities of occurrence of each PL state in each of the 4 experimental conditions for the optimal solution with $\mathrm{k}=7$ are shown in the bar plots of Figure 6B. As predicted from the previous analysis, we find that for $k=7$, psilocybin significantly alters the occurrence of two of the seven BOLD phase-locking modes. The probability of occurrence of PL state 3 (red) significantly decreased from $14.3 \pm 2.4 \%$ pre-injection (third most visited state) to $4.1 \pm 1.2 \%$ following the psilocybin administration ( $p=0.00034$ uncorrected, $p^{\prime}=0.0021$ after correction). Conversely, a significant increase in the probability of occurrence of PL state 1 (dark blue) was also observed, shifting from $26.7 \pm 4.5 \%$ pre-psilocybin injection to $37.2 \pm 6.0 \%$ post-psilocybin injection $(p=$ 
0.0068 uncorrected, $p^{\prime}=0.047$ after correction). In contrast, the probability of occurrence of all other PL states remained statistically similar before and after the psilocybin infusion (all p-values $>0.05)$.

\section{Validation using the placebo dataset}

The 7 PL states shown in Figure 2 were defined solely from half of the fMRI scans in the dataset, i.e., the scans from the 9 participants recorded during the day in which psilocybin was infused

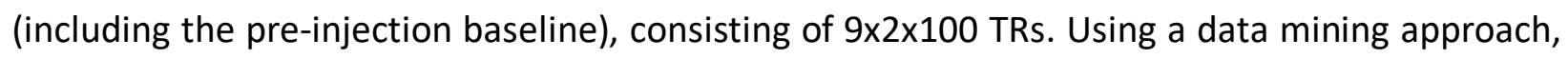
we searched for the partition model that returned the most significant differences between preand post- injection conditions.

To further validate our findings, we verified whether these same 7 PL states were expressed in the $\mathrm{fMRI}$ session during which placebo was infused - recorded more than a week apart -, and if they occurred with similar probabilities as during the baseline condition before psilocybin was injected. To do so, we took exactly the same cluster centroids (sown in Figure 2A) and evaluated their probabilities of occurrence both before and after the placebo infusion.

Validating our results, we found that the probabilities of occurrence of all $7 \mathrm{PL}$ states both before and after placebo injection did not differ from the baseline condition before psilocybin was infused (all p-values > 0.05). Notably, this indicates that the dynamical exploration of PL states assessed with LEiDA remained stable across subjects under baseline conditions over a period of more than a week (Figure 6B). Corroborating our previous findings, the fractional occupancy of PL state 3 remained within normal levels both before $(15.0 \pm 3.3 \%)$ and after $(14.3 \pm 2.9 \%)$ placebo injection ( $p=0.49$ uncorrected), and was in each case significantly higher than the probability of occurrence found during the psychedelic state $(4.1 \pm 1.2 \%, p=0.00057$ and $p=$ 0.00014 , respectively, both falling below the corrected threshold $\left.\alpha_{2}=0.05 / k=0.007\right)$. Moreover, the probability of occurrence of PL state 1, remained unchanged following the placebo injection $(23.6 \pm 5.6 \%$ versus $27.4 \pm 3.1 \%, p=0.38)$, being substantially lower than the probability of occurrence of PL state 1 under psilocybin $\left(37.2 \pm 6.0 \%, p=0.00051\right.$ before, $p^{\prime}=0.012$ after correction) placebo injection, with the pre-placebo comparison surviving correction for multiple comparisons with $\alpha_{2}$. 

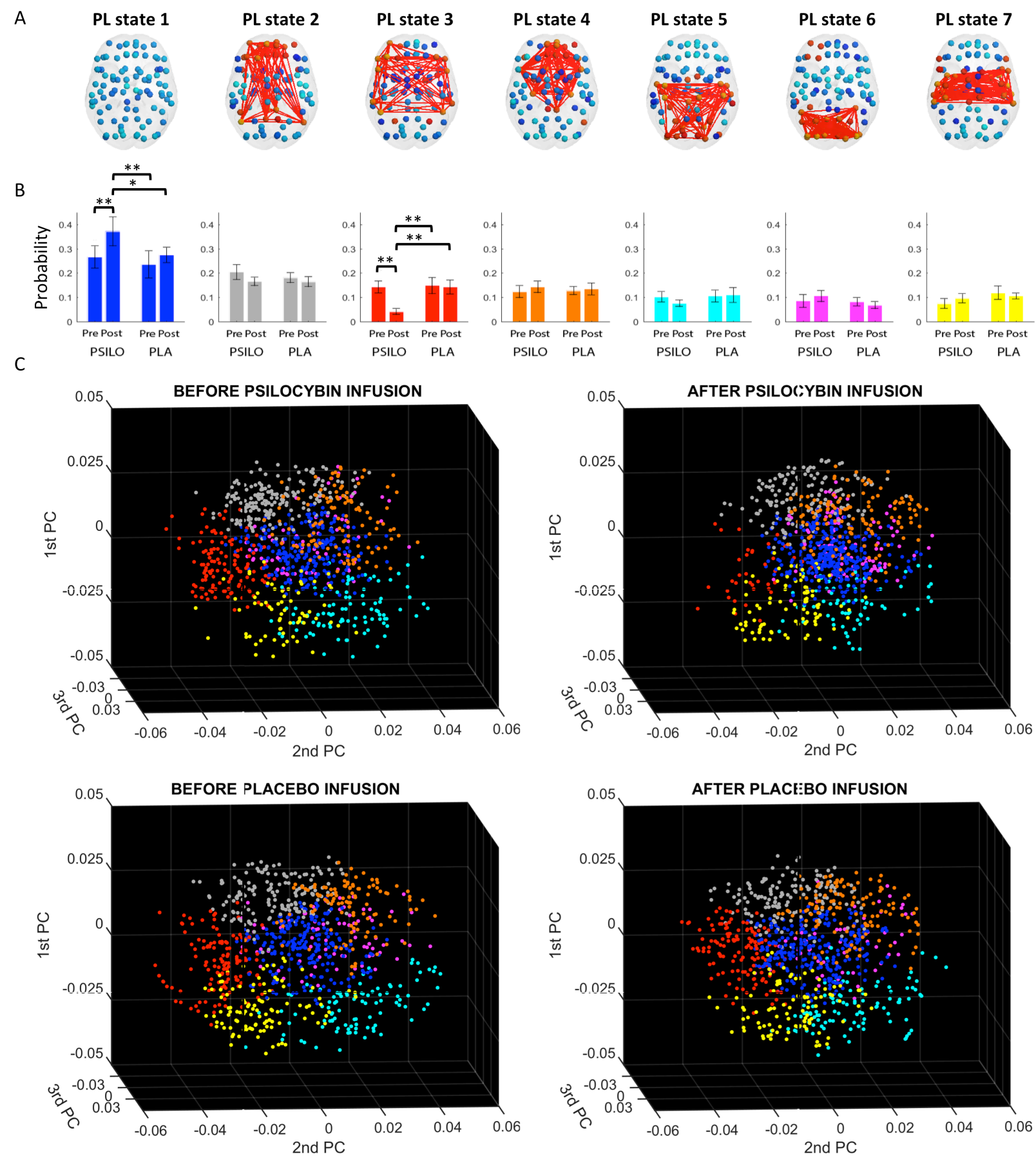

Figure 6 - Psilocybin modulates the exploration of BOLD Phase-Locking configurations. A - Repertoire of PL states detected with LEiDA for $\mathrm{k}=7$ from the session when psilocybin was infused (same as shown in Figure 2). B - Probability of occurrence (mean \pm standard error of the mean across subjects) of each PL state in each of the four experimental conditions (pre/post psilocybin and pre/post placebo injection). We find that after the infusion of psilocybin, PL state 3 (red) significantly decreases in probability of occurence from an average of $14.3 \%$ to $4.1 \%(p=0.00034)$, whereas PL state 1 (blue) significantly increases after psilocybin injection ( $p=0.0068)$. All other PL states did not change in fractional occupancy with psilocybin. 
Importantly, when the BOLD phase leading eigenvectors from the placebo session (900 before and 900 after infusion) were matched to the $7 \mathrm{PL}$ cluster centroids obtained from the psilocybin session, we found that all the probabilities of occurrence were consistent with the resting state before psilocybin injection (despite being more than a week apart), and exhibited the same significant differences with respect to the psilocybin condition. Asterisks denote the presence of statistically significant differences $(*<0.05 ; * *$ $<0.05 / \mathrm{k}$ correcting for the number of independent hypothesis tested). C - Representation of all PL configurations in each experimental condition on a low-dimensional manifold highlighting the reduced number of occurrences of the Frontoparietal network (red dots) after psilocybin injection. In each scatter plot, each dot represents one PL configuration, captured by $V_{1}(t)$. Each panel corresponds to a different session (before/after psilocybin/placebo). Dots are colored according to the closest cluster centroid vector, $\mathrm{V}_{\mathrm{C}}$, using the same color code as in $\mathrm{B}$. It can clearly be observed that the number of red dots is substantially reduced after the psilocybin injection. This representation serves to illustrate how the reduced occurrence of the Frontoparietal network under psilocybin can be interpreted in terms of fewer excursions into a specific region of a low-dimensional manifold of network configurations.

\section{Dynamical exploration in a low-dimensional manifold}

The non-stationary dynamics of the PL leading eigenvectors over time can be interpreted as the constant exploration of a cloud of PL configurations in a multi-dimensional space (with $\mathrm{N}=90$ dimensions in the selected parcellation). In order to visualize this cloud of observations in a lowdimensional manifold (Figure 6C), we applied a Principal Components Analysis (PCA) to all leading eigenvectors across conditions and subjects $(90 \times 3600)$ and used the first 3 Principal Components $(3 \times 3600)$ to project each observation in a low-dimensional manifold. In this way, each 90 dimensional leading eigenvector $V_{1}$ obtained at each TR can be plotted as a dot in a 3-dimensional (3-D) space defined by the 3 first principal components and colored according to its closest centroid vector, $V_{c}$, using the same color scheme from Figure 6B. By construction, the k-means clustering algorithm produces compact clusters (i.e., non-overlapping and well separated in the 90 dimensional space where the leading eigenvectors are defined). As can be seen in Figure 6C, retaining the first 3 principal components is sufficient to conserve well-defined clusters (see also Supplementary Figure S3 to view the 3-D manifolds from a different angle).

This representation serves to illustrate how the occurrence of the different functional networks can be interpreted mechanistically in terms of excursions into distinct regions of a lowdimensional manifold of network configurations. The visual rendition of the data from all subjects in the 4 different experimental conditions shown in Figure $6 \mathrm{C}$ highlights the reduced number of excursions into the region of the manifold associated to the frontoparietal PL state under psilocybin (number of red dots in the upper right-hand plot), compared to the 3 non-psychedelic conditions (before psilocybin and before/after placebo injection). Although less evident, it can also be seen that the blue cluster, corresponding to the globally coherent PL state 1, becomes denser (more dots) after the psilocybin injection. The statistical analysis shown in Figure $5 \mathrm{C}$ serves to define the partition model that optimally delineates the region of the cloud affected after psilocybin injection. 


\section{Trajectories between BOLD phase-locking states}

In order to explore the trajectories between the different PL states, we report in Figure 7 the switching matrices containing the mean probabilities of, being in a given PL state (rows), transitioning to any of the other PL states (columns) both before (left) and after the psilocybin injection (right). Switching probabilities were calculated for each subject and each condition and then statistically compared using a permutation-based paired t-test (5000 permutations). To facilitate the interpretation of the differences found in PL state switching profiles, we provide an illustration of the transitions that were most significantly affected by psilocybin (solid red: increased; dashed blue: decreased). We find that the probability of transitioning from any given PL state to the frontoparietal PL state 3 is consistently reduced under psilocybin effects. Conversely, all PL states except one were more likely to transition toward the PL state of global coherence following the psilocybin injection. The $p$-values for each state-to-state transition in the switching matrix are provided in Supplementary Table ST1. 

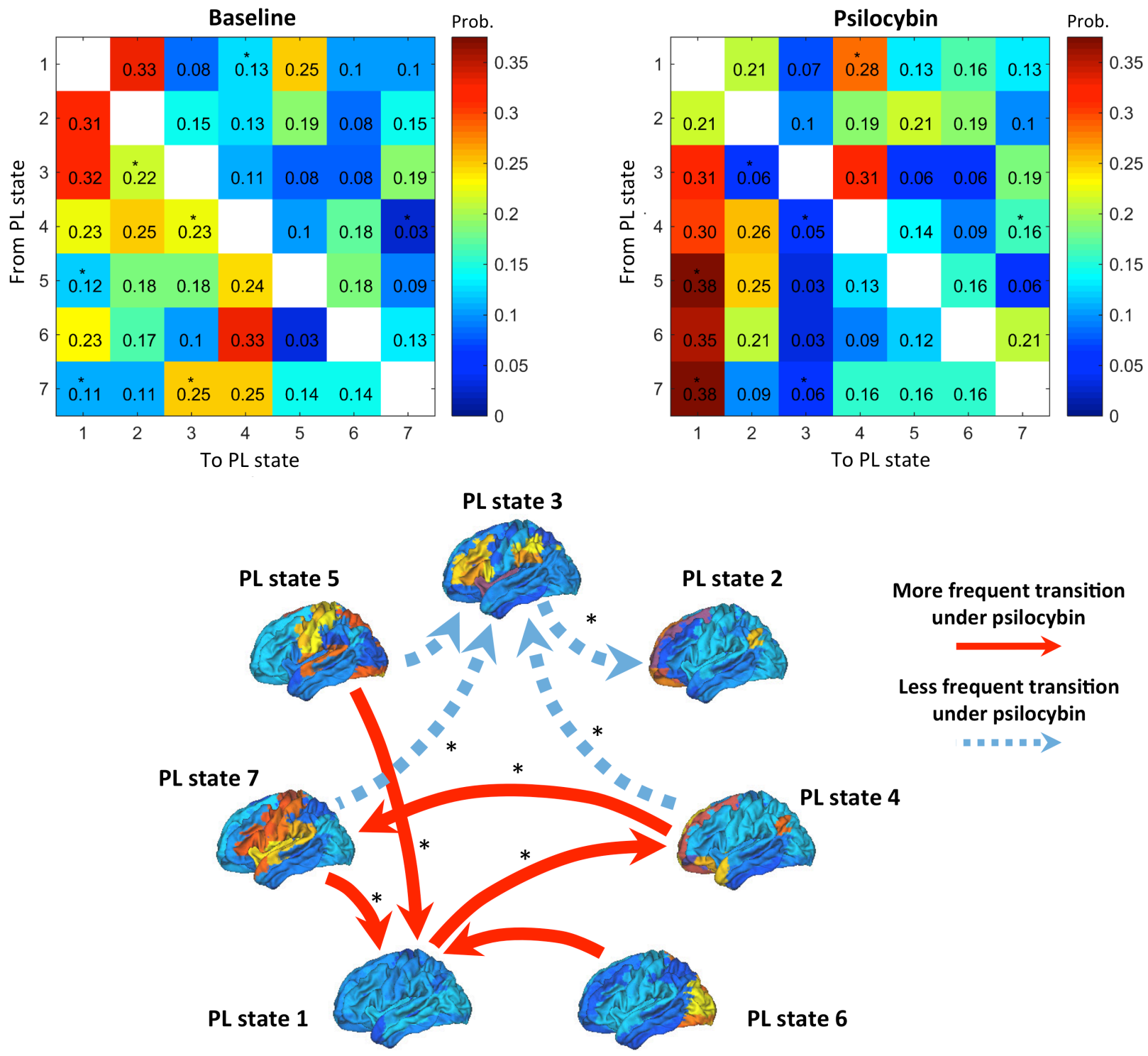

Figure 7-Psilocybin modifies the switching patterns between PL states. Top: Switching matrices showing the probability of, being in a given PL state (rows), transitioning to any of the other PL states (columns) both before (left) and after the psilocybin injection (right). Significant between-condition differences assessed via a permutation test are indicated by asterisks $\left({ }^{*}\right)$ for the significance threshold $\alpha_{1}=0.05$. Bottom: Pre vs post-injection changes in the transition probabilities between PL states (rendered on the cortical surface for illustration purposes). Each arrow represents a state-to-state transition that increased (solid red) or decreased (dashed blue) more than one standard deviation in probability after psilocybin injection (asterisks denote significance with $p<0.05$ ). This representation illustrates how some transitions to (and from) the frontoparietal PL state 3 became less frequent after psilocybin injection. Conversely, transitions toward the globally coherent PL state 1 became more frequent following the psilocybin injection. 


\section{Subjective intensity of the psychedelic experience correlates with network occupancy}

After each scanning session participants were asked to rate the subjective intensity of the psychedelic (or placebo) experience on a scale of 1 to 10 . We correlated those subjective intensity ratings with the probability of occurrence of each of the $7 \mathrm{PL}$ states (Figure 8). We found that the occurrence of frontoparietal network (PL state 3) was negatively correlated with the subjective rating of the psychedelic experience (Pearson's $r=0.56 ; p=0.0083$, one-tailed). Conversely, a near-significant positive correlation was found between the fractional occupancy of the globally coherent PL state 1 and the subjective intensity of the psychedelic experience $(r=0.36, p=0.07$, one-tailed). Other PL states whose fractional occupancy was not changed by psilocybin did not correlate with this behavioral measure.
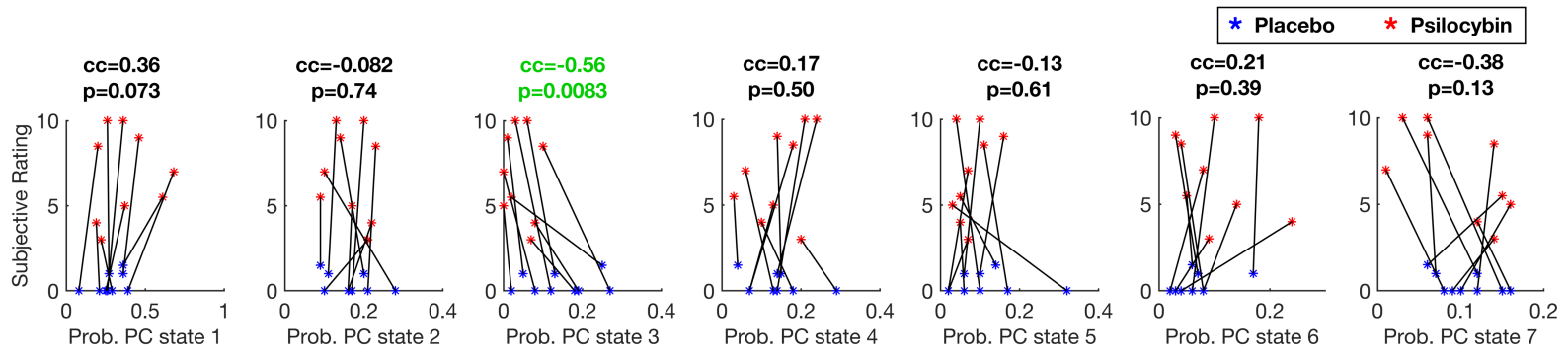

Figure 8 - Subjective intensity rating of the psychedelic experience is related to dynamic exploration of PL states. After the infusion of either Psilocybin (red) or Placebo (blue), participants were asked to rate the subjective intensity of their experience which in each case was far stronger in the psilocybin condition (mean intensity $=6.9 \pm 2.6$ ) than placebo (mean intensity $=0.4 \pm 0.6$ ) on a scale from 0 to 10 . We further investigated the relationship between the subjective ratings and the probability of occurrence of each of the 7 PL states. We found that the occurrence of the frontoparietal network (PL state 3 ) was negatively correlated with the ratings on psychedelic experience (correlation coefficient $c c=0.56 ; p=0.0083$, onetailed), with higher intensity ratings being associated with a lower probability of occurrence of PL state 3 (black lines link the same participant in the placebo (blue) and psilocybin (red) conditions). Conversely, a near-significant positive correlation was found between the fractional occupancy of the globally coherent PL state 1 and the subjective intensity of the psychedelic experience ( $c c=0.36, p=0.073$, one-tailed).

\section{Psilocybin modulates the global order of BOLD phases}

We also investigated how psilocybin affected the global order of BOLD phases using the Kuramoto Order Parameter (OP) and, although the mean synchrony degree did not reveal significant differences between conditions ( $p>0.05)$, we found that the degree of whole-brain metastability (i.e., how much the synchrony degree fluctuates over time) significantly increased following the psilocybin injection compared to baseline (standard deviation, $\operatorname{STD}(O P)=0.18 \pm$ 0.01 vs $\mathrm{STD}(\mathrm{OP})=0.16 \pm 0.015, \mathrm{t}(8)=2.41, \mathrm{p}=0.04$ using a paired sample $\mathrm{t}$-test). In Supplementary Figure S4, we report the OP over time before and after the psilocybin injection in all subjects.

In addition, we investigated how the OP relates with the different PL states. As expected, we found that the probability of occurrence of the PL state of global coherence (state 1 ) at the single subject level during a given scan is strongly correlated with the mean of the global OP ( $c c=0.825$, $p=2.9 * 10^{-5}$ ) (Supplementary Figure S4), indicating that this PL state is associated with an increase in global order, whereas all other PL states are consistently found for epochs of lower OP. In 
Supplementary Figure S4, we report the global OP obtained while each of the 7 PL states was active, according to the color-code used in Figure 6B. This allows a visual representation of all PL state assignments in all subjects at the single TR resolution, showing that PL state 1 is the most frequently visited state - both before and after the psilocybin injection - and also occurs in epochs of high OP, indicating a high level of phase coherence across the network nodes.

Finally, we ran a paired-sample t-test to confirm that, at the individual subject level, the mean number of occurrences of PL state 3 (overlapping with to the Frontoparietal network) was significantly decreased following the psilocybin injection relative to the pre-injection baseline from $14.3 \pm 2.5 \%$ to $4.1 \pm 1.2 \%(t(8)=3.88, p=0.005)$. Conversely, a paired-sample t-test showed a significant increase in the mean number of occurrences of PL state 1 (globally coherent state) following the psilocybin injection relative to the pre-injection baseline from $26 . \% \pm 4.5 \%$ to 37.2 $\pm 6.0 \%(t(8)=2.75, p=0.01)$. These statistical results reinforce the validity of the findings reported in Figure 6B.

\section{Effect of motion on PL state probabilities}

We performed follow-up analyses to confirm that the observed effects of psilocybin on the fractional occupancy of PL state 1 and PL state 3 were not due to differences in participant motion during the scan. For the nine subjects included in the study, we assessed motion by calculating the mean framewise displacement (FD) for each subject. This variable measures movement of any given frame relative to the previous frame. We then conducted two separate analyses to examine the potential effects of motion. First, a paired t-test showed that the mean FD was not significantly different between the post-placebo and post-psilocybin injection condition: $t(8)=$ 1.28, $p=0.24$. Furthermore, the fractional occupancy of PL state 3 post-psilocybin did not correlate with the mean FD (correlation coefficient, $C C=-0.14, p=0.72$ ) and neither did the fractional occupancy of PL state 1 post-psilocybin ( $C C=0.04, p=0.91$ ).

\section{Discussion}

The present study is one of the first to investigate how the exploration of the brain's repertoire of functional networks is rapidly modulated by a psychoactive molecule, here the serotonergic psychedelic psilocybin. Specifically, we found that the dynamical exploration of a fixed repertoire of discrete functional networks at rest, defined as recurrent BOLD phase-locking patterns over time (PL states), is significantly altered after a psilocybin infusion. The strongly reduced expression of a BOLD PL state overlapping with the previously described frontoparietal control system (Vincent et al., 2008, Yeo et al., 2011) suggests that this particular functional subsystem is related to the psychoactive effects of psilocybin. Moreover, we found that the decreased expression of the Frontoparietal network under the influence of the drug only increased the stability of a state of global coherence (PL state 1) and did not change the expression of the remaining PL patterns. These new findings represent one of the first attempts at bridging the gap between molecular pharmacodynamics and the brain's network dynamics. 
The highly significant disruption of a BOLD PL pattern closely overlapping with the frontoparietal control system is consistent with prior human neuroimaging studies of psilocybin's effects. Indeed, a previous analysis of the same dataset revealed that psilocybin modified the BOLD spectral content in a distributed frontoparietal network. Specifically, the frontoparietal system of interest showed significantly decreased power at low frequencies (and decreased power spectrum scaling exponent) under psilocybin (Tagliazucchi et al., 2014). Similarly, an MEG study reported decreased oscillatory power under psilocybin in a bilateral frontoparietal network derived from beta-band activity (Muthukumaraswamy et al., 2013). Findings from these prior studies indicate a rapid desynchronization of the frontoparietal control system under psilocybin and are thus consistent with the present results. Furthermore, the disengagement of the frontoparietal system can be interpreted in light of the cognitive and behavioral effects of psilocybin reported by the study participants. Evidence suggests the frontoparietal system plays a key role in cognitive control by focusing attention on goal-relevant information through topdown mechanisms (Cole and Schneider, 2007, Dosenbach et al., 2008, Xin and Lei, 2015). Such goal-directed focus does indeed appear to be impaired under moderate-high doses of psilocybin (Bayne and Carter, 2018) - which would be a reasonable way of describing the present dosage. Declines in frontoparietal FC have been associated with difficulty suppressing goal-irrelevant information, and greater distractibility (Smallwood et al., 2012). These observations are broadly consistent with the reports of increased freely wandering thoughts and hyper-associative cognition reported by participants in the present cohort (Carhart-Harris et al., 2012, Turton et al., 2014).

It is interesting to consider how the known pharmacodynamic properties of psilocybin could mechanistically explain its destabilizing effect on the frontoparietal PL state dynamics described above. The psychoactive effects of psilocybin are primarily due to its agonist activity at the serotonin 2A (5- $\mathrm{HT}_{2 \mathrm{~A}}$ ) receptor (Vollenweider et al., 1998, Kometer et al., 2013). The distribution and density of $5-\mathrm{HT}_{2 \mathrm{~A}}$ receptors in the human brain (and other 5-HT receptor subtypes) were recently estimated in a high-resolution atlas using molecular and structural neuroimaging data in a large cohort of healthy individuals in combination with postmortem human brain autoradiography (Beliveau et al., 2017); $5-\mathrm{HT}_{2 \mathrm{~A}}$ receptors are widely distributed across the brain but most densely expressed in the cortex. Interestingly, the $5-\mathrm{HT}_{2 \mathrm{~A}}$ receptor density map obtained by Beliveau and colleagues shows a strong qualitative overlap between 5$\mathrm{HT}_{2 \mathrm{~A}}$ receptor expression on the lateral surface of the brain, and the regions implicated in the frontoparietal PL state detected in the present study. Moreover, an fMRI study of the synthetic $5-\mathrm{HT}_{2 \mathrm{~A}}$ receptor agonist and classical psychedelic lysergic acid diethylamide (LSD) has reported significantly increased functional connectivity density (FCD, quantified as the average functional connectivity to the rest of the brain) of a similar frontoparietal network which, in turn, strongly overlapped with 5- $\mathrm{HT}_{2 \mathrm{~A}}$ receptor concentrations (Tagliazucchi et al., 2016). The increase in FCD of the Frontoparietal network can be interpreted as reduced within-network integrity and is thus consistent with the present findings, where the PL state 3 (high within-network coherence and 
low coherence with the rest of the brain, equivalent to lower FCD) is replaced by the globally coherent PL state 1 (equivalent to higher FCD).

At the cellular level, studies have shown that $5-\mathrm{HT}_{2 \mathrm{~A}}$ receptors are highly expressed in layer 5 pyramidal neurons (Andrade and Weber, 2010). Stimulation of $5-\mathrm{HT}_{2 \mathrm{~A}}$ receptors induces a slow depolarization of pyramidal cells via G-protein coupled signaling pathways as well as the inhibition of the calcium-activated after-hyperpolarization (Andrade, 2011); together these changes make these neurons more likely to fire. Our results are therefore consistent with a scenario in which psilocybin increases the excitability of layer 5 pyramidal neurons broadly in the brain, wherever they are expressed. However, if the expression of $5-\mathrm{HT}_{2 \mathrm{~A}}$ receptors is especially high in lateral frontoparietal areas, then the dysregulated excitation that follows their stimulation (Carhart-Harris, 2019) may, in turn, destabilize this particular system, weakening its stability within the brain's broader repertoire. Supporting this idea, a recent modeling study showed that while the brain's structural connectome plays an important role in constraining and predicting brain dynamics, an additional layer of explanatory power is offered by considering energetic constraints on those dynamics (Gu et al., 2018). Because maintaining functional connections is energetically costly, the brain normally favors efficient neural codes and wiring patterns (Attwell and Laughlin, 2001, Bullmore and Sporns, 2012, Lord et al., 2013). In this framework, the metabolic costs of establishing and maintaining functional connections between anatomically distributed neurons in this Frontoparietal network may become too high following the psilocybin infusion, and brain activity might spend less time in this particular state as a result.

Consistent with this explanation, our analysis revealed increased stability of a globally coherent state (PL state 1) following the psilocybin injection. Previous work has indeed shown that the serotonergic psychedelics psilocybin and LSD induce an alternative type of functional integration, characterized by greater global integration (Petri et al., 2014, Roseman et al., 2014, Tagliazucchi et al., 2016) as well as increases in the metastability of brain states (Carhart-Harris et al., 2014). The results presented here not only align with these prior findings but also shed further light on their functional significance. Here we found that a globally coherent state, i.e., PL state 1, increased its predominance under psilocybin, not only statistically over time, but also dynamically, as revealed by the state-to-state switching matrix, where it became the 'state of choice' for inter-state transitions. In other words, under psilocybin the brain spent more time in a globally coherent, highly integrated state. This state therefore served as the dominant attractor, absorbing the majority of inter-state transitions. As was implied by previous analyses, this effect may enable atypical patterns of interregional communication to arise in the psychedelic state (Petri et al., 2014) alongside the breakdown of within-network integrity in favor of globally integrated brain dynamics (Muthukumaraswamy et al., 2013, Carhart-Harris et al., 2014, Petri et al., 2014, Roseman et al., 2014, Tagliazucchi et al., 2016).

In addition to changing the relative stability of PL states and the directionality of state transitions, we found that psilocybin increased the global metastability of brain dynamics. This result is consistent with prior fMRI investigations of the same psilocybin dataset in which a 
greater diversity of functional connectivity motifs was observed after psilocybin infusion within a small network consisting of the bilateral hippocampal and anterior cingulate brain regions, thus reflecting increased variability in the collective repertoire of metastable states (Tagliazucchi et al., 2014). This prior analysis had purposely limited the number of regions included in order to perform an exhaustive counting of all possible functional connectivity states. Another study had found increased metastability in several canonical resting-state networks as determined by the variance in the networks' intrinsic synchrony over time (relationship between the mean and variance of the signal over time) (Carhart-Harris et al., 2014). Here we used a complementary analytical approach to demonstrate that increased metastability of BOLD phase coherence under psilocybin is generalizable at the whole-brain scale by calculating the standard deviation of the order parameter of the system over time.

Despite the fact that there were only 9 subjects included in this fMRI dataset, which is a relatively small number to ensure statistical power, our findings are strongly validated by the replication of results in placebo conditions across the two scanning days (i.e., no significant changes in the occupancy of network states in the placebo scanning session vs pre-psilocybin infusion), as well as the strong statistical effect in specific PL states after psilocybin injection (with $p<0.0005$, surviving correction for the number of independent hypothesis tested). Importantly, since LEiDA compares the statistics between a discrete number of whole-brain PL patterns rather than individual functional links, the number of independent hypothesis tested is reduced from $\mathrm{N}(\mathrm{N}-1) / 2$ (number of unique links in a NxN symmetric matrix) to the number of states $\mathrm{K}$, which overcomes several of the statistical limitations associated with multiple comparisons. Furthermore, given the instantaneous nature of LEiDA, we detected significant betweencondition differences despite the fact that each scanning condition consisted only of $100 \mathrm{fMRI}$ volumes, which would likely not have been sufficient for conventional sliding-window analysis (Hindriks et al., 2015). Moreover, we have demonstrated that the changes in the fractional occupancy of specific PL states after the psilocybin infusion was unrelated to participant motion during the scan.

The significant overlap between the BOLD phase-locking patterns detected automatically with LEiDA (from just $1800 \mathrm{fMRI}$ volumes on 9 subjects) and the canonical resting-state networks obtained from ICA analysis on >1000 subjects (Yeo et al., 2011) suggests that the relative phase of the BOLD signals is sensitive to the same underlying patterns captured using ICA procedures, with the advantage that the networks can have spatial overlap and occur transiently and at different frames in time. Although our approach did not reveal all the 7 RSNs reported in Yeo et al. (2011) - for instance, none of our PL states revealed the Dorsal Attention Network - this difference could be due to our limited sample size. Further investigations on a larger sample size are likely to provide additional insights on the mechanisms underlying the formation of these BOLD phase-locking patterns and their relation to well-established RSNs. Importantly, using the cosine function, we focus on phase-locked oscillatory states and do not consider any directed causal influence between BOLD signals, which is fundamentally different to approaches 
considering asymmetric connectivity measures (Gilson et al., 2016). Regarding the temporal dynamics, we note that the LEiDA approach allows for the analysis of additional temporal properties of BOLD phase-locking patterns, including the dwell time of each state, as in Cabral et al. (2017b) and more recently in Figueroa et al. (2019). However, the temporal resolution used in the current dataset ( $T R=3$ seconds) was too slow to capture more rapid dynamics, which are suggested by MEG studies to operate at time scales of approximately 200ms (Baker et al., 2014, Vidaurre et al., 2016). As such, we limited our study to the probabilities of occurrence and to the transition profiles occurring over these longer time scales.

The therapeutic potential of psilocybin in psychiatry has recently generated much interest. Indications of efficacy have been reported for conditions including treatment-resistant depression, anxiety related to end-of-life care and addictive disorders (Griffiths et al., 2006, Grob et al., 2011, Johnson et al., 2014, Carhart-Harris et al., 2016). The neural mechanisms underlying these clinical benefits, however, remain unclear. The present results provide some of the first evidence that a psychoactive compound modulates the brain's dynamical repertoire by selectively destabilizing a brain functional network (i.e., frontoparietal control system) and promoting transitions towards a globally coherent PL state. We believe this represents an exciting first step towards bridging molecular pharmacodynamics and dynamical measures of brain function in macro-scale networks, not only for serotonergic psychedelics but potentially across other classes of drugs and neuromodulators. This could have exciting implications for the design of novel therapeutics for neuropsychiatric disorders informed by patho-connectomics, which may be generally understood in terms of targeting the specific PL states affected by a particular disorder with neuromodulatory approaches. Moreover, despite the small sample size and the use of a coarse behavioral measure, we were nevertheless able to show strong associations between the fractional occupancy of certain PL states and the subjective intensity of the psychedelic experience. This interesting proof of concept represents, to our knowledge, one of the first attempts at linking molecular pharmacodynamics, dynamical brain measures and behaviour. We hope this work paves the way for future work in this exciting direction.

In summary, the present study used a novel, data-driven dynamical functional connectivity analysis (LEiDA) to investigate how psilocybin rapidly modulates the exploration of the brain's repertoire of functional network states at rest. We found that a PL state closely corresponding to a canonical frontoparietal control system was destabilized by the compound, while transitions toward a globally coherent PL state were enhanced. We also found an increase in the metastability of global brain dynamics following the psilocybin infusion. Taken together, these findings are consistent with prior neuroimaging studies suggesting that a different type of brain integration and increased neural signal complexity underlie the psychedelic state. The present results also suggest that characterizing neuromodulatory effects on the brain's dynamical repertoire may help guide circuit-specific pharmacological interventions in neuropsychiatric disorders. 


\section{Acknowledgments}

LDL is supported by the Canadian Institutes of Health Research, Canadian Centennial Scholarship Fund and the Mann Senior Scholarship from Hertford College, University of Oxford. MLK is supported by the ERC Consolidator Grant CAREGIVING (n. 615539) and Center for Music in the Brain, funded by the Danish National Research Foundation (DNRF117). JC is supported under the project FronThera NORTE-01-0145-FEDER-000023 from the Northern Portugal Regional Operational Programme (NORTE 2020) under the Portugal 2020 Partnership Agreement through the European Regional Development Fund (FEDER). RCH is supported by the Alex Mosley Charitable Trust, Ad Astra Chandaria Foundation and Tamas Foundation. The authors are grateful to the Beckley Foundation for funding the original research study, on which Amanda Feilding, Director of the Beckley Foundation, was a vital initiator and collaborative partner.

\section{References}

Acebrón JA, Bonilla LL, Vicente CJP, Ritort F, Spigler R (2005) The Kuramoto model: A simple paradigm for synchronization phenomena. Reviews of modern physics 77:137.

Acebron JA, Perales A, Spigler R (2001) Bifurcations and global stability of synchronized stationary states in the Kuramoto model for oscillator populations. Physical review E, Statistical, nonlinear, and soft matter physics 64:016218.

Andrade R (2011) Serotonergic regulation of neuronal excitability in the prefrontal cortex. Neuropharmacology 61:382-386.

Andrade R, Weber ET (2010) Htr2a gene and 5-HT2A receptor expression in the cerebral cortex studied using genetically modified mice. Frontiers in neuroscience 4:36.

Attwell D, Laughlin SB (2001) An energy budget for signaling in the grey matter of the brain. Journal of Cerebral Blood Flow \& Metabolism 21:1133-1145.

Baker AP, Brookes MJ, Rezek IA, Smith SM, Behrens T, Smith PJP, Woolrich M (2014) Fast transient networks in spontaneous human brain activity. elife 3.

Bayne T, Carter O (2018) Dimensions of consciousness and the psychedelic state. Neuroscience of consciousness 2018:niy008.

Beckmann CF, DeLuca M, Devlin JT, Smith SM (2005) Investigations into resting-state connectivity using independent component analysis. Philosophical transactions of the Royal Society of London Series B, Biological sciences 360:1001-1013.

Beckmann CF, Smith SM (2004) Probabilistic independent component analysis for functional magnetic resonance imaging. IEEE transactions on medical imaging 23:137-152.

Beliveau V, Ganz M, Feng L, Ozenne B, Højgaard L, Fisher PM, Svarer C, Greve DN, Knudsen GM (2017) A high-resolution in vivo atlas of the human brain's serotonin system. Journal of Neuroscience 37:120-128.

Biswal B, Zerrin Yetkin F, Haughton VM, Hyde JS (1995) Functional connectivity in the motor cortex of resting human brain using echo-planar MRI. Magnetic resonance in medicine 34:537-541.

Bressler SL, Menon V (2010) Large-scale brain networks in cognition: emerging methods and principles. Trends in cognitive sciences 14:277-290.

Brookes MJ, Hale JR, Zumer JM, Stevenson CM, Francis ST, Barnes GR, Owen JP, Morris PG, Nagarajan SS (2011) Measuring functional connectivity using MEG: methodology and comparison with fcMRI. Neurolmage 56:1082-1104. 
Bullmore E, Sporns O (2012) The economy of brain network organization. Nature Reviews Neuroscience 13:336.

Cabral J, Hugues E, Sporns O, Deco G (2011) Role of local network oscillations in resting-state functional connectivity. Neuroimage 57:130-139.

Cabral J, Kringelbach M, Deco G (2017a) Functional Connectivity dynamically evolves on multiple timescales over a static Structural Connectome: Models and Mechanisms. Neurolmage.

Cabral J, Kringelbach ML, Deco G (2014a) Exploring the network dynamics underlying brain activity during rest. Progress in neurobiology 114:102-131.

Cabral J, Luckhoo H, Woolrich M, Joensson M, Mohseni H, Baker A, Kringelbach ML, Deco G (2014b) Exploring mechanisms of spontaneous functional connectivity in MEG: how delayed network interactions lead to structured amplitude envelopes of band-pass filtered oscillations. Neurolmage 90:423-435.

Cabral J, Vidaurre D, Marques P, Magalhaes R, Silva Moreira P, Miguel Soares J, Deco G, Sousa N, Kringelbach ML (2017b) Cognitive performance in healthy older adults relates to spontaneous switching between states of functional connectivity during rest. Scientific reports 7:5135.

Carhart-Harris RL (2019) How do psychedelics work? Current opinion in psychiatry 32:16-21.

Carhart-Harris RL, Bolstridge M, Rucker J, Day CM, Erritzoe D, Kaelen M, Bloomfield M, Rickard JA, Forbes $B$, Feilding A (2016) Psilocybin with psychological support for treatment-resistant depression: an open-label feasibility study. The Lancet Psychiatry 3:619-627.

Carhart-Harris RL, Erritzoe D, Williams T, Stone JM, Reed L, Colasanti A, Tyacke RJ, Leech R, Malizia AL, Murphy K (2012) Neural correlates of the psychedelic state as determined by fMRI studies with psilocybin. Proceedings of the National Academy of Sciences 109:2138-2143.

Carhart-Harris RL, Leech R, Hellyer PJ, Shanahan M, Feilding A, Tagliazucchi E, Chialvo DR, Nutt D (2014) The entropic brain: a theory of conscious states informed by neuroimaging research with psychedelic drugs. Frontiers in human neuroscience 8.

Cavanna F, Vilas MG, Palmucci M, Tagliazucchi E (2017) Dynamic functional connectivity and brain metastability during altered states of consciousness. Neurolmage.

Christoff K, Irving ZC, Fox KC, Spreng RN, Andrews-Hanna JR (2016) Mind-wandering as spontaneous thought: a dynamic framework. Nature reviews Neuroscience 17:718-731.

Cole MW, Schneider W (2007) The cognitive control network: integrated cortical regions with dissociable functions. Neuroimage 37:343-360.

Damoiseaux JS, Rombouts SA, Barkhof F, Scheltens P, Stam CJ, Smith SM, Beckmann CF (2006) Consistent resting-state networks across healthy subjects. Proceedings of the National Academy of Sciences of the United States of America 103:13848-13853.

De Luca M, Beckmann CF, De Stefano N, Matthews PM, Smith SM (2006) fMRI resting state networks define distinct modes of long-distance interactions in the human brain. Neurolmage 29:13591367.

Deco G, Jirsa V, McIntosh AR, Sporns O, Kotter R (2009) Key role of coupling, delay, and noise in resting brain fluctuations. Proceedings of the National Academy of Sciences of the United States of America 106:10302-10307.

Deco G, Kringelbach M (2016) Metastability and Coherence: Extending the Communication through Coherence Hypothesis Using a Whole-Brain Computational Perspective. Trends in neurosciences 39:432.

Dosenbach NU, Fair DA, Cohen AL, Schlaggar BL, Petersen SE (2008) A dual-networks architecture of topdown control. Trends in cognitive sciences 12:99-105. 
Figueroa CA, Cabral J, Mocking RJT, Rapuano KM, van Hartevelt TJ, Deco G, Expert P, Schene AH, Kringelbach ML, Ruhe HG (2019) Altered ability to access a clinically relevant control network in patients remitted from major depressive disorder. Human brain mapping.

Gilson M, Moreno-Bote R, Ponce-Alvarez A, Ritter P, Deco G (2016) Estimation of Directed Effective Connectivity from fMRI Functional Connectivity Hints at Asymmetries of Cortical Connectome. PLoS computational biology 12:e1004762.

Glerean E, Salmi J, Lahnakoski JM, Jaaskelainen IP, Sams M (2012) Functional magnetic resonance imaging phase synchronization as a measure of dynamic functional connectivity. Brain connectivity 2:91101.

González-Maeso J, Weisstaub NV, Zhou M, Chan P, Ivic L, Ang R, Lira A, Bradley-Moore M, Ge Y, Zhou Q (2007) Hallucinogens recruit specific cortical 5-HT 2A receptor-mediated signaling pathways to affect behavior. Neuron 53:439-452.

Griffiths RR, Richards WA, McCann U, Jesse R (2006) Psilocybin can occasion mystical-type experiences having substantial and sustained personal meaning and spiritual significance. Psychopharmacology 187:268-283.

Grob CS, Danforth AL, Chopra GS, Hagerty M, McKay CR, Halberstadt AL, Greer GR (2011) Pilot study of psilocybin treatment for anxiety in patients with advanced-stage cancer. Archives of general psychiatry 68:71-78.

Gu S, Cieslak M, Baird B, Muldoon SF, Grafton ST, Pasqualetti F, Bassett DS (2018) The energy landscape of neurophysiological activity implicit in brain network structure. Scientific reports 8:2507.

Gutierrez-Barragan D, Basson MA, Panzeri S, Gozzi A (2018) Oscillatory brain states govern spontaneous fMRI network dynamics. bioRxiv 393389.

Hansen EC, Battaglia D, Spiegler A, Deco G, Jirsa VK (2015) Functional connectivity dynamics: modeling the switching behavior of the resting state. Neuroimage 105:525-535.

Hellyer PJ, Scott G, Shanahan M, Sharp DJ, Leech R (2015) Cognitive Flexibility through Metastable Neural Dynamics Is Disrupted by Damage to the Structural Connectome. The Journal of neuroscience : the official journal of the Society for Neuroscience 35:9050-9063.

Hindriks R, Adhikari MH, Murayama Y, Ganzetti M, Mantini D, Logothetis NK, Deco G (2015) Can slidingwindow correlations reveal dynamic functional connectivity in resting-state fMRI? Neurolmage 127:242-256.

Hipp JF, Hawellek DJ, Corbetta M, Siegel M, Engel AK (2012) Large-scale cortical correlation structure of spontaneous oscillatory activity. Nature neuroscience 15:884-890.

Jenkinson M, Bannister P, Brady M, Smith S (2002) Improved optimization for the robust and accurate linear registration and motion correction of brain images. Neuroimage 17:825-841.

Johnson MW, Garcia-Romeu A, Cosimano MP, Griffiths RR (2014) Pilot study of the 5-HT2AR agonist psilocybin in the treatment of tobacco addiction. Journal of psychopharmacology 28:983-992.

Karahanoğlu FI, Van De Ville D (2015) Transient brain activity disentangles fMRI resting-state dynamics in terms of spatially and temporally overlapping networks. Nature communications 6:7751.

Kometer M, Schmidt A, Jäncke L, Vollenweider FX (2013) Activation of serotonin 2A receptors underlies the psilocybin-induced effects on $\alpha$ oscillations, N170 visual-evoked potentials, and visual hallucinations. Journal of Neuroscience 33:10544-10551.

Lempel A, Ziv J (1976) On the complexity of finite sequences. IEEE Transactions on information theory 22:75-81.

Liu X, Duyn JH (2013) Time-varying functional network information extracted from brief instances of spontaneous brain activity. Proceedings of the National Academy of Sciences of the United States of America 110:4392-4397. 
Lord L-D, Expert P, Huckins JF, Turkheimer FE (2013) Cerebral energy metabolism and the brain's functional network architecture: an integrative review. Journal of Cerebral Blood Flow \& Metabolism 33:1347-1354.

Lord L-D, Stevner AB, Deco G, Kringelbach ML (2017) Understanding principles of integration and segregation using whole-brain computational connectomics: implications for neuropsychiatric disorders. Philosophical Transactions of the Royal Society A: Mathematical, Physical and Engineering Sciences 375.

Mantini D, Perrucci MG, Del Gratta C, Romani GL, Corbetta M (2007) Electrophysiological signatures of resting state networks in the human brain. Proceedings of the National Academy of Sciences of the United States of America 104:13170-13175.

McKenna DJ, Repke DB, Lo L, Peroutka SJ (1990) Differential interactions of indolealkylamines with 5hydroxytryptamine receptor subtypes. Neuropharmacology 29:193-198.

Meltzer H, Massey B, Horiguchi M (2012) Serotonin receptors as targets for drugs useful to treat psychosis and cognitive impairment in schizophrenia. Current pharmaceutical biotechnology 13:1572-1586.

Menon V (2011) Large-scale brain networks and psychopathology: a unifying triple network model. Trends in cognitive sciences 15:483-506.

Musso F, Brinkmeyer J, Mobascher A, Warbrick T, Winterer G (2010) Spontaneous brain activity and EEG microstates. A novel EEG/fMRI analysis approach to explore resting-state networks. Neurolmage 52:1149-1161.

Muthukumaraswamy SD, Carhart-Harris RL, Moran RJ, Brookes MJ, Williams TM, Errtizoe D, Sessa B, Papadopoulos A, Bolstridge M, Singh KD (2013) Broadband cortical desynchronization underlies the human psychedelic state. Journal of Neuroscience 33:15171-15183.

Newman ME (2006) Finding community structure in networks using the eigenvectors of matrices. Physical review $E$, Statistical, nonlinear, and soft matter physics 74:036104.

Passie T, Seifert J, Schneider U, Emrich HM (2002) The pharmacology of psilocybin. Addiction biology 7:357-364.

Petri G, Expert P, Turkheimer F, Carhart-Harris R, Nutt D, Hellyer PJ, Vaccarino F (2014) Homological scaffolds of brain functional networks. Journal of The Royal Society Interface 11:20140873.

Ponce-Alvarez A, Deco G, Hagmann P, Romani GL, Mantini D, Corbetta M (2015) Resting-state temporal synchronization networks emerge from connectivity topology and heterogeneity. PLoS computational biology 11:e1004100.

Roseman L, Leech R, Feilding A, Nutt DJ, Carhart-Harris RL (2014) The effects of psilocybin and MDMA on between-network resting state functional connectivity in healthy volunteers. Frontiers in human neuroscience 8:204.

Schartner MM, Carhart-Harris RL, Barrett AB, Seth AK, Muthukumaraswamy SD (2017) Increased spontaneous MEG signal diversity for psychoactive doses of ketamine, LSD and psilocybin. Scientific Reports 7.

Seeley WW, Menon V, Schatzberg AF, Keller J, Glover GH, Kenna H, Reiss AL, Greicius MD (2007) Dissociable intrinsic connectivity networks for salience processing and executive control. The Journal of neuroscience : the official journal of the Society for Neuroscience 27:2349-2356.

Shanahan M (2010) Metastable chimera states in community-structured oscillator networks. Chaos 20:013108.

Smallwood J, Brown K, Baird B, Schooler JW (2012) Cooperation between the default mode network and the frontal-parietal network in the production of an internal train of thought. Brain research 1428:60-70.

Smith SM (2002) Fast robust automated brain extraction. Human brain mapping 17:143-155. 
Tagliazucchi E, Balenzuela P, Fraiman D, Chialvo DR (2012) Criticality in large-scale brain FMRI dynamics unveiled by a novel point process analysis. Frontiers in physiology 3:15.

Tagliazucchi E, Carhart-Harris R, Leech R, Nutt D, Chialvo DR (2014) Enhanced repertoire of brain dynamical states during the psychedelic experience. Human brain mapping 35:5442-5456.

Tagliazucchi E, Roseman L, Kaelen M, Orban C, Muthukumaraswamy SD, Murphy K, Laufs H, Leech R, McGonigle J, Crossley N (2016) Increased global functional connectivity correlates with LSDinduced ego dissolution. Current Biology 26:1043-1050.

Tewarie P, Abeysuriya R, Byrne Á, O'Neill GC, Sotiropoulos SN, Brookes MJ, Coombes S (2018) How do spatially distinct frequency specific MEG networks emerge from one underlying structural connectome? The role of the structural eigenmodes. Neurolmage.

Tononi G, Edelman GM (1998) Consciousness and complexity. Science 282:1846-1851.

Turton S, Nutt D, Carhart-Harris R (2014) A qualitative report on the subjective experience of intravenous psilocybin administered in an FMRI environment. Current drug abuse reviews 7:117-127.

Tzourio-Mazoyer N, Landeau B, Papathanassiou D, Crivello F, Etard O, Delcroix N, Mazoyer B, Joliot M (2002) Automated anatomical labeling of activations in SPM using a macroscopic anatomical parcellation of the MNI MRI single-subject brain. Neurolmage 15:273-289.

Varela FJ (1979) Principles of biological autonomy.

Vidaurre D, Quinn AJ, Baker AP, Dupret D, Tejero-Cantero A, Woolrich MW (2016) Spectrally resolved fast transient brain states in electrophysiological data. Neurolmage 126:81-95.

Vidaurre D, Smith SM, Woolrich MW (2017) Brain network dynamics are hierarchically organized in time. Proceedings of the National Academy of Sciences 114:12827-12832.

Vincent JL, Kahn I, Snyder AZ, Raichle ME, Buckner RL (2008) Evidence for a frontoparietal control system revealed by intrinsic functional connectivity. Journal of neurophysiology 100:3328-3342.

Vollenweider FX, Vollenweider-Scherpenhuyzen MF, Bäbler A, Vogel H, Hell D (1998) Psilocybin induces schizophrenia-like psychosis in humans via a serotonin-2 agonist action. Neuroreport 9:38973902.

Watanabe T, Hirose S, Wada H, Imai Y, Machida T, Shirouzu I, Konishi S, Miyashita Y, Masuda N (2014) Energy landscapes of resting-state brain networks. Frontiers in neuroinformatics 8:12.

Xin F, Lei X (2015) Competition between frontoparietal control and default networks supports social working memory and empathy. Social cognitive and affective neuroscience 10:1144-1152.

Yeo BT, Krienen FM, Sepulcre J, Sabuncu MR, Lashkari D, Hollinshead M, Roffman JL, Smoller JW, Zöllei L, Polimeni JR (2011) The organization of the human cerebral cortex estimated by intrinsic functional connectivity. Journal of neurophysiology 106:1125. 